\title{
A Monolithic CMOS Autocompensated Sensor Transducer for Capacitive Measuring Systems
}

\author{
Cheng-Ta Chiang, Member, IEEE, Chi-Shen Wang, and Yu-Chung Huang
}

\begin{abstract}
In this paper, a monolithic complimentary metaloxide-semiconductor (CMOS) autocompensated sensor transducer for capacitive measuring systems is newly presented. The proposed converter is compact and robust to integrate in capacitive measuring systems. The proposed autocompensated sensor transducer is attractive due to the fact that a digitized signal is produced without realizing the analog-to-digital converter. Hence, the hardware cost could be reduced. Furthermore, the output signal of the proposed transducer is a pulse stream; it could be easily sent over a wide range of transmission media, such as package switch networks (PSNs), radios, and optical, infrared (IR), and ultrasonic media. Another innovation is that the proposed automatic compensation circuits enhance and compensate the linear relation between the variable capacitance of the detected sensor and the output digital frequency over a wide dynamic frequency range. Measurement results have successfully verified the functions and the performance of the proposed autocompensated sensor transducer and confirmed that it is possible to apply it to the air pressure sensor. The area of this chip is $940 \times 1080 \mu \mathrm{m}^{2}$, and the power consumption is $6.4 \mathrm{~mW}$. The proposed transducer is not only suitable for capacitive measuring systems but also practical for application in the front-end systems of the wireless sensor network.
\end{abstract}

Index Terms-Capacitive sensor, complimentary metal-oxidesemiconductor (CMOS), sensor interface, sensor transducer, signal conditioning.

\section{INTRODUCTION}

$\mathbf{R}$ ECENTLY, sensor transducers have been an attractive topic of research for sensor measuring instruments and automotive and medical systems. For example, these transducers have been suitably used in pressure transducers [1], [2], humidity sensing systems [3], [4], etc. To fit the demands of the commercial market on sensor transducers, the proposed autocompensated sensor transducer, which is implemented as a capacitance-to-frequency converter (CFC), has the feature

Manuscript received May 30, 2007; revised November 3, 2007. First published June 10, 2008; current version published October 10, 2008. This work was supported by the National Science Council of Taiwan, R.O.C., under Contract NSC92-2218-E-009-011.

C.-T. Chiang is with Mixed Signal Design Technologies Division, SoC Technology Center, Industrial Technology Research Institute, Hsinchu 310, Taiwan, R.O.C., and also with the Measurement Techniques Laboratory, Department of Electronics Engineering and Institute of Electronics, National Chiao Tung University, Hsinchu 300, Taiwan, R.O.C. (e-mail: ctchiang23.ee90g@ nctu.edu.tw).

C.-S. Wang is with Mobile Devices Incorporation, Hsinchu, Taiwan, R.O.C., and also with the Measurement Techniques Laboratory, Department of Electronics Engineering and Institute of Electronics, National Chiao Tung University, Hsinchu 300, Taiwan, R.O.C.

Y.-C. Huang is with the Measurement Techniques Laboratory, Department of Electronics Engineering and Institute of Electronics, National Chiao Tung University, Hsinchu 300, Taiwan, R.O.C.

Color versions of one or more of the figures in this paper are available online at http://ieeexplore.ieee.org.

Digital Object Identifier 10.1109/TIM.2008.925020
TABLE I

COMPARISONS to Previous InTEgrated SENSOR TRANSDUCERS

\begin{tabular}{|c|c|c|c|}
\hline & [7] & [8] & This work \\
\hline Technology & NMOS $5 \mu \mathrm{m}$ & CMOS $4 \mu \mathrm{m}$ & CMOS $0.35 \mu \mathrm{m}$ \\
\hline Power supply & $10 \mathrm{~V}$ & $\pm 1.5 \mathrm{~V}$ & $3.2 \mathrm{~V}$ \\
\hline $\begin{array}{c}\text { Input Capacitance } \\
\text { range }\end{array}$ & $20-50 \mathrm{pF}$ & $2-20 \mathrm{pF}$ & $4-24 \mathrm{pF}$ \\
\hline $\begin{array}{c}\text { Maximum } \\
\text { Frequency range }\end{array}$ & $1.2 \mathrm{kHz}$ & $1 \mathrm{kHz}$ & $500 \mathrm{kHz}$ \\
\hline Chip area & $2.4 \mathrm{~mm}^{2}$ & $1.1 \mathrm{~mm}^{2}$ & $1.01 \mathrm{~mm}^{2}$ \\
\hline
\end{tabular}

of being low cost. The proposed autocompensated sensor transducer is attractive due to the fact that a digitized signal is produced without realizing the analog-to-digital converter. Thus, the hardware cost could be reduced. Furthermore, the output signal of the proposed transducer is a pulse stream; it could be easily sent over a wide range of transmission media, such as package switch networks, radios, and optical, infrared, and ultrasonic media. Hence, the output signal could easily be received and processed by the receiver terminal, such as the receiver in the systems of a wireless sensor network. Until now, several achievements [5]-[8] had been realized. However, the output frequency of these transducers could not be higher than a 100-kHz frequency band, as listed in Table I. Hence, they are not suitable for present applications, such as demanding a wide dynamic frequency range. To satisfy such sensor requirements, a novel design with digitized compensation circuits is, thus, investigated first [9].

In this paper, a monolithic CMOS autocompensated sensor transducer for capacitive measuring systems is newly proposed. The proposed converter is compact and robust for integration into capacitive measuring systems. Based on the $0.35-\mu \mathrm{m}$ $2 \mathrm{P} 4 \mathrm{M}$ CMOS technology with a $3.2-\mathrm{V}$ power supply, all the functions and the performance of the proposed converter are tested and proven through Simulation Program with Integrated Circuit Emphasis (SPICE) simulations. Measurement results have successfully verified the functions and the performance of the proposed autocompensated sensor transducer and confirmed that it is possible to apply them to the air pressure sensor. The output frequency range is from 0.5 to $500 \mathrm{kHz}$, and the variable capacitance of the detected sensor ranges from 4 to $24 \mathrm{pF}$. The area of this chip is $940 \times 1080 \mu \mathrm{m}^{2}$, and the power consumption is $6.4 \mathrm{~mW}$. The proposed transducer is not only suitable for capacitive measuring systems but is also practical for application in the front-end systems of the wireless sensor network.

In Section II, the capacitive sensing principle of the air pressure sensor is addressed. The system architecture and 


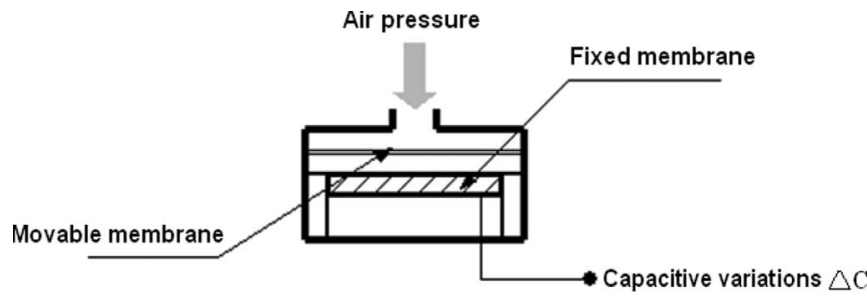

Fig. 1. Structure of the air pressure sensor.

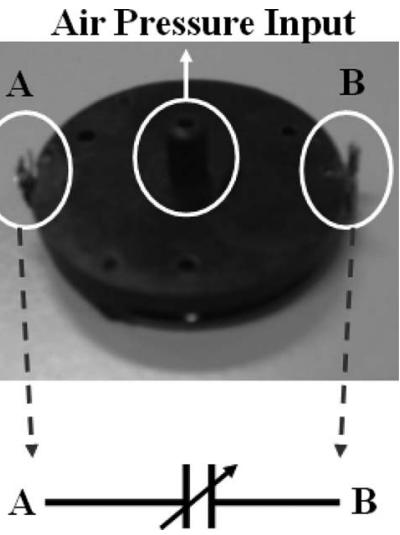

Fig. 2. Physical structure of the air pressure sensor.

simulation results are described in Section III. Section IV displays the measurement results. Last, Section V provides the conclusions and future works.

\section{Capacitive Sensing Principle of THE AIR PRESSURE SENSOR}

First, the capacitive sensing principle of the air pressure sensor is addressed. The structure of the air pressure sensor is shown in Fig. 1. The controlled air pressure passes through a channel and pushes the movable membrane. When the movable membrane starts to shift, the distance between the fixed and movable membranes is changed. Thus, the capacitive variations $\Delta C$ can be derived as

$$
\begin{aligned}
C & =\varepsilon \frac{A}{d} \\
\Delta C & =\frac{A}{d} \Delta \varepsilon-\frac{\varepsilon A}{d^{2}} \Delta d+\frac{\varepsilon}{d} \Delta A .
\end{aligned}
$$

Combining (1) and (2)

$$
\frac{\Delta C}{C}=\frac{\Delta \varepsilon}{\varepsilon}-\frac{\Delta d}{d}+\frac{\Delta A}{A}
$$

where $\varepsilon$ is the dielectric constant, $A$ is the area of two metal plates, and $d$ is the gap distance. In the air pressure sensor, the parameters of $\varepsilon$ and $A$ will not be changed. Thus, the capacitive variations $\Delta C$ will be directly affected by the gap distance $\Delta d$. By controlling the air pressure, the capacitive variations of the air pressure sensor can be obtained by (3). Fig. 2 shows the physical structure of the air pressure sensor, and the experimental characteristics are measured in Fig. 3. In Section IV, the air pressure sensor will be applied and experimented on within the proposed autocompensated sensor transducer.

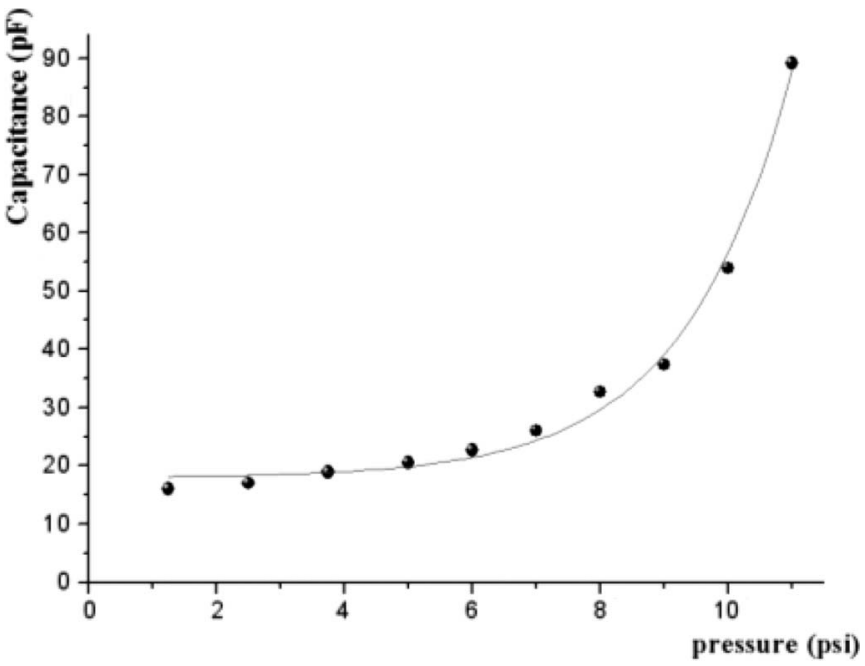

Fig. 3. Experimental characteristic of the air pressure sensor.

\section{System Architecture and Simulation Results}

Now, the system architecture and circuits are analyzed. Fig. 4 shows the schematic of the proposed autocompensated sensor transducer, which consists of the capacitance-to-voltage converter (CVC), the voltage-to-frequency converter (VFC), and the automatic compensation circuits. By following Section III, the configurations, the operation principles of all the function blocks, and the compensation algorithm of the automatic compensation circuits will be described in detail.

\section{A. $C V C$}

Among the proposed CFC, the CVC needs to be particularly taken care of. That is because this interfacing circuit is first contacted to the detected sensor. Furthermore, the offset voltage of the operational amplifier (OP) and the charge injection error of a switch should be particularly considered. The schematics of the CVC with the OP offset and switch charge injection insensitive properties are shown in Fig. 5. $C_{x}$ is the capacitor of the detected sensor, and $C_{R}$ and $C_{F}$ are the designed capacitors. $V_{R 1}$ is the common-mode voltage $(1.6 \mathrm{~V})$, and $V_{R 2}$ is the reference voltage $(2.1 \mathrm{~V})$. The signals $c k_{1}$ and $c k_{2}$ are two nonoverlapping phase clocks. When the signal $c k_{2}$ is logic high, the voltage $V_{R 2}$ will charge the capacitor $C_{x}$, whereas the capacitor $C_{F}$ stores the offset voltage of the OP. The MOS $M_{5}$ is switched on, and the output voltage is $V_{R 1}$. When the signal $c k_{1}$ is logic high, the capacitor $C_{F}$ is connected to the output. The voltage $V_{R 2}$ charges the capacitor $C_{R}$. Thus, by following the principle of charge conservation, the output voltage $V_{o}$ will be derived as follows:

$$
\begin{aligned}
& C_{x}\left(V_{R 2}-V_{R 1}-V_{o s}\right)+C_{R}\left(0-V_{o s}\right)+C_{F}\left(0-V_{o s}\right) \\
& \quad=C_{x}\left(0-V_{o s}\right)+C_{R}\left(V_{R 2}-V_{R 1}-V_{o s}\right)+C_{F}\left(V_{o}-V_{o s}\right) .
\end{aligned}
$$

Therefore

$$
V_{o}=\frac{C_{x}-C_{R}}{C_{F}}\left(V_{R 2}-V_{R 1}\right)
$$




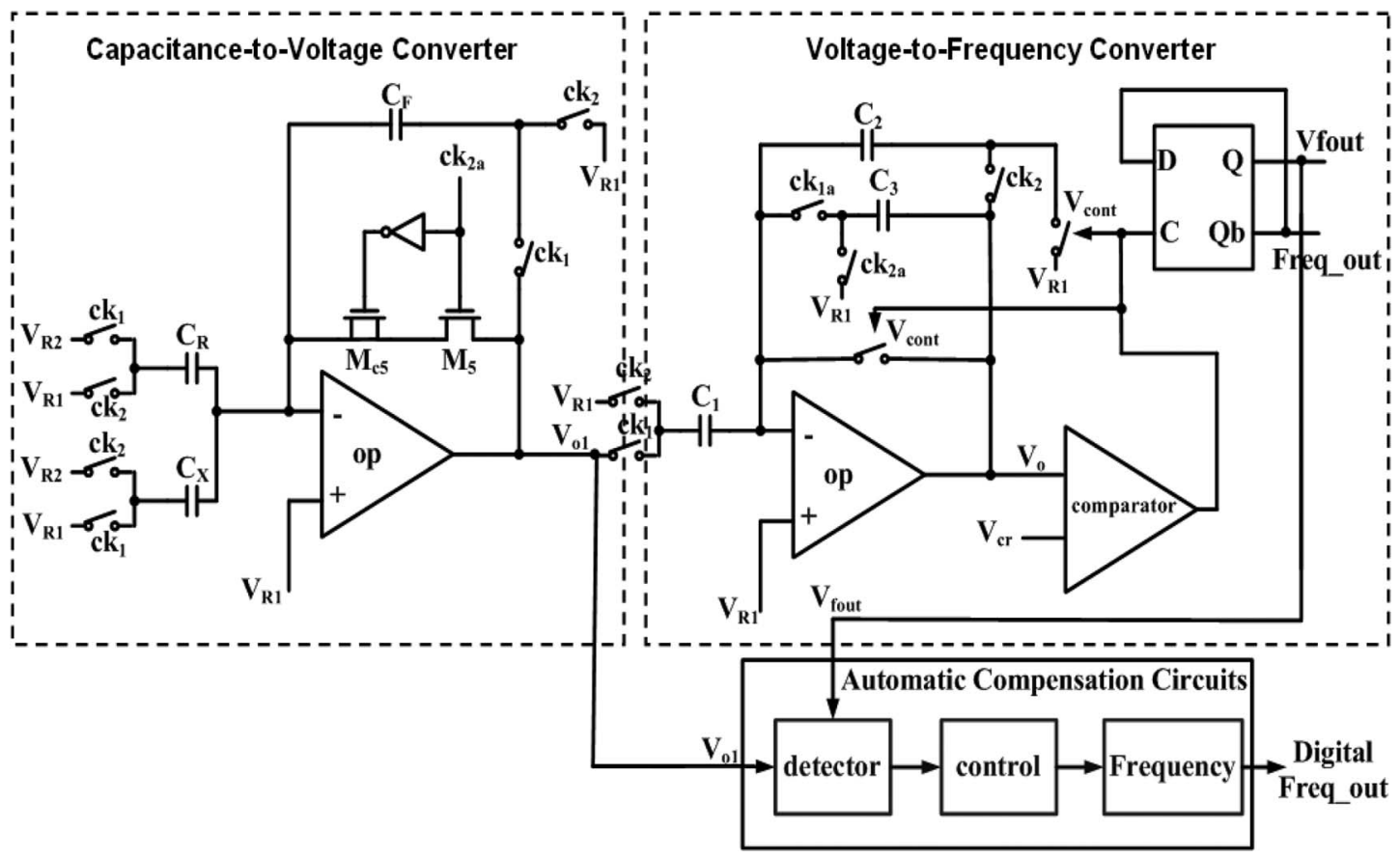

Fig. 4. Schematic of the proposed autocompensated sensor transducer.

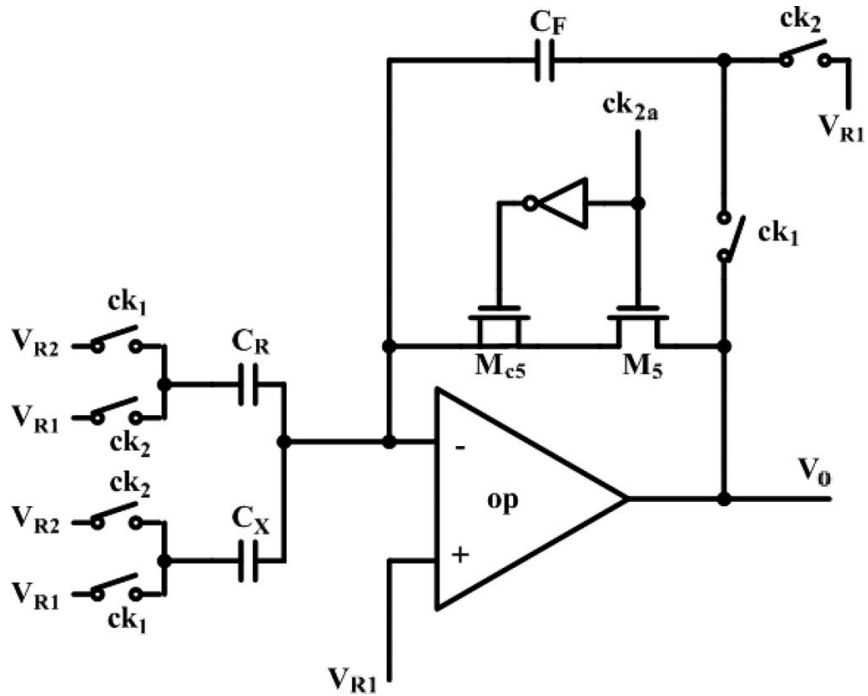

Fig. 5. Schematic of the CVC with the OP offset and switch charge injection insensitive properties.

By following (5), the OP offset can be removed. Thus, the CVC with the OP offset insensitive property is verified. Next, the charge injection error of a switch is to be discussed. The voltage error is generated when the MOS $M_{5}$ is turned off. The produced charge injection error $\Delta V_{e}$ that is contributed to the output node will be given as follows:

$$
\Delta V_{e}=\frac{C_{g s 5} * V_{c k 2 a}}{C_{F}}
$$

To solve this error, the MOS $M_{c 5}$ is designed to absorb the charge injection from the MOS $M_{5} . c k_{2 a}$ is the clock signal that is raised slightly before the signal $c k_{2}$. When the signal $c k_{2 a}$ goes from logic high to logic low, the MOS $M_{5}$ will be turned off. When the signal $c k_{2 a}$ is from logic high to logic

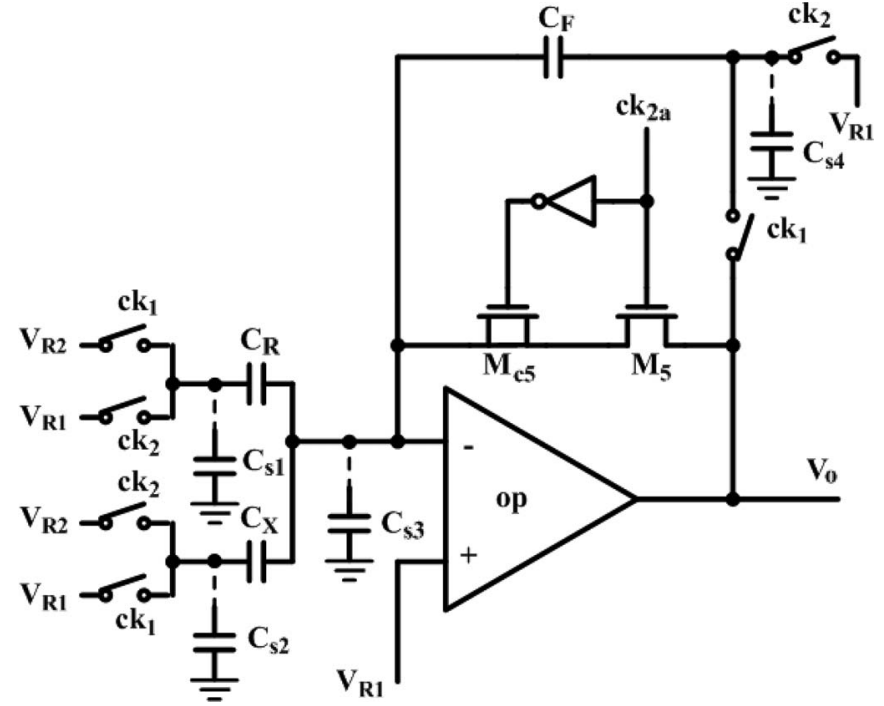

Fig. 6. Schematic of the CVC with parasitic capacitors $C_{s 1}$ to $C_{s 4}$.

low, the MOS $M_{c 5}$ turns on and absorbs the charge from the MOS $M_{5}$. Thus, the problem of the charge injection error can be overcome.

Moreover, the OP plays an important role in the converter. Therefore, to discuss the effect of the OP nonideality, the OP specification must be addressed. The setting time and the resolution are all dependent on the OP gain and bandwidth. All the OP considerations are discussed below. At the beginning, the OP gain is derived as

$$
\begin{aligned}
C_{x}\left(V_{R 2}-V_{R 1}\right)= & C_{x}\left(V_{R 1}-\mathrm{OP}_{-}\right)+C_{R}\left(V_{R 2}-\mathrm{OP}_{-}\right) \\
& +C_{F}\left(V_{o}-\mathrm{OP}_{-}\right) \\
V_{R 1}-\mathrm{OP}_{-}= & \frac{V_{o}}{A}, \quad A: \text { Gain. }
\end{aligned}
$$




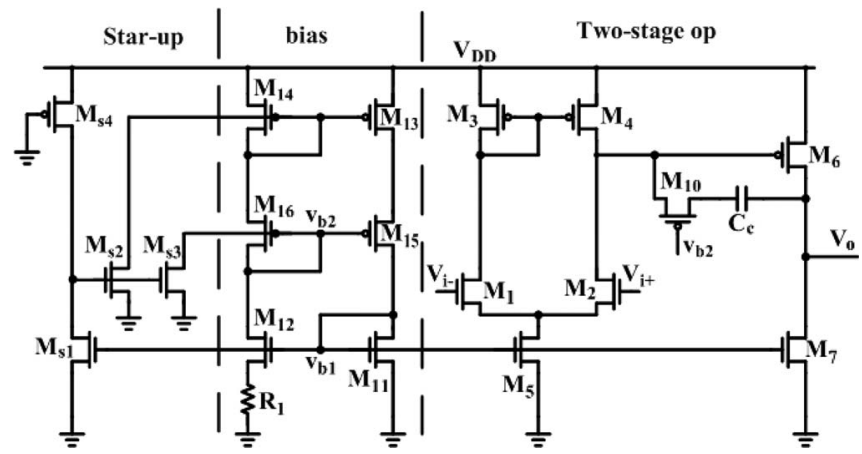

Fig. 7. Schematic of the OP.

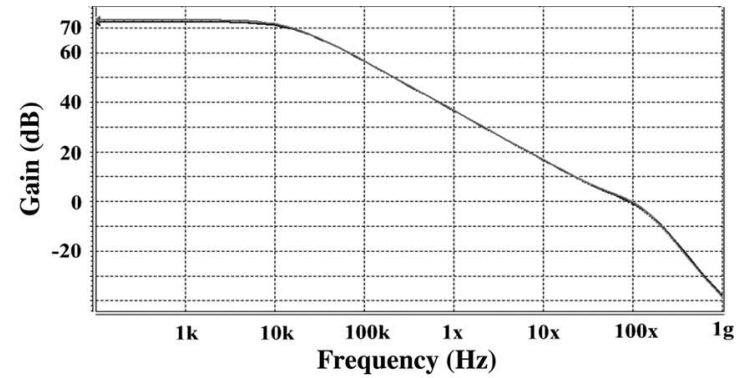

(a)

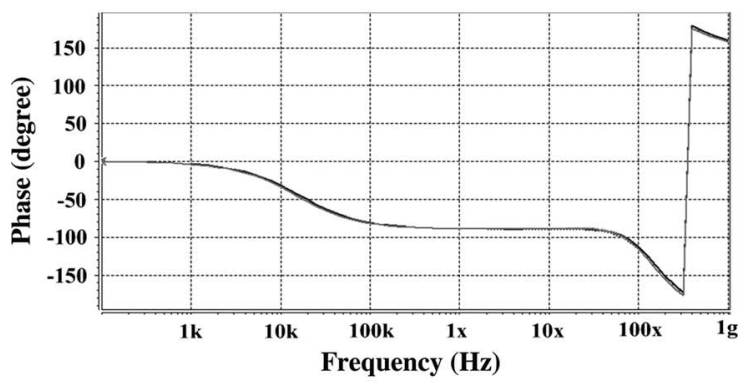

(b)

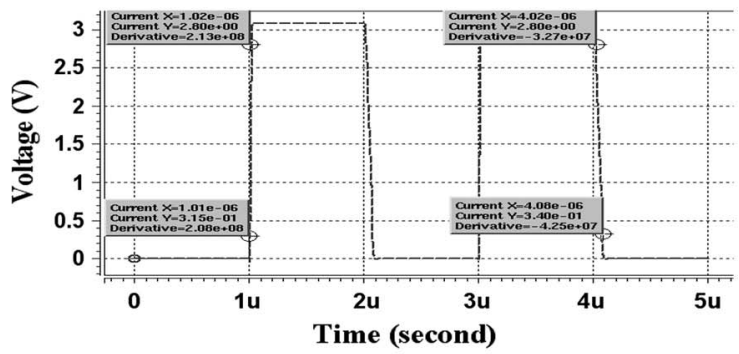

(c)

Fig. 8. SPICE simulation results of the OP under frequency sweeping from $0.1 \mathrm{~Hz}$ to $1 \mathrm{GHz}$. (a) Gain. (b) Phase. (c) SR response.

Therefore

$$
\begin{aligned}
V_{o} & =\frac{\left(C_{x}-C_{R}\right)}{C_{F}}\left(V_{R 2}-V_{R 1}\right) *\left(\frac{1}{1+\frac{1}{A}\left(\frac{C_{F}+C_{R}+C_{x}}{C_{F}}\right)}\right) \\
& =\frac{\left(C_{x}-C_{R}\right)}{C_{F}}\left(V_{R 2}-V_{R 1}\right) *\left(1-\frac{1}{A\left(\frac{C_{F}}{C_{F}+C_{R}+C_{x}}\right)}\right)
\end{aligned}
$$

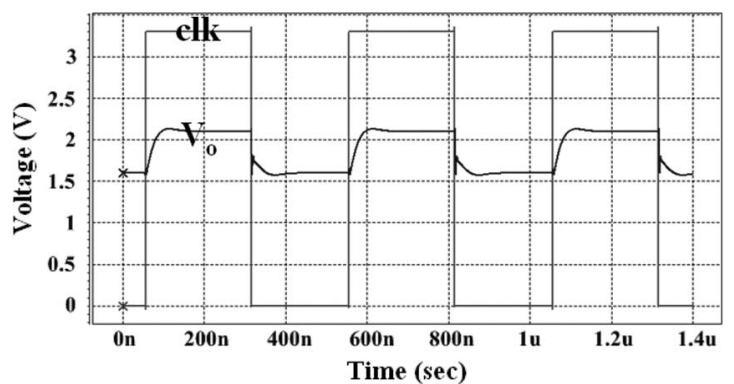

(a)

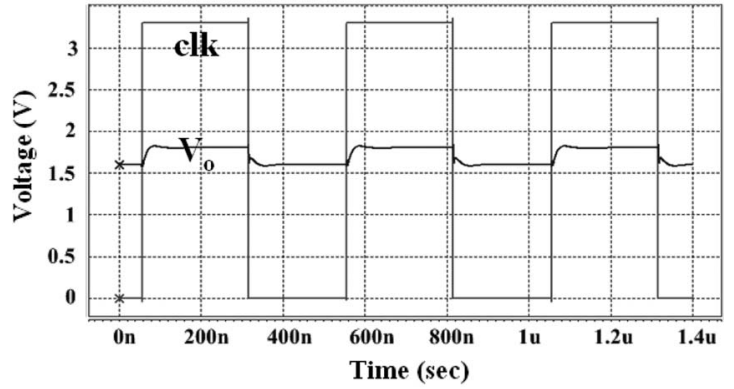

(b)

Fig. 9. SPICE output waveforms under the capacitance $C_{F}=20 \mathrm{pF}$ and $C_{R}=4 \mathrm{pF}$, the voltages $V_{R 1}=1.6 \mathrm{~V}$ and $V_{R 2}=2.1 \mathrm{~V}$, and the capacitance $C_{x}$ of (a) $24 \mathrm{pF}$ and (b) $12 \mathrm{pF}$.

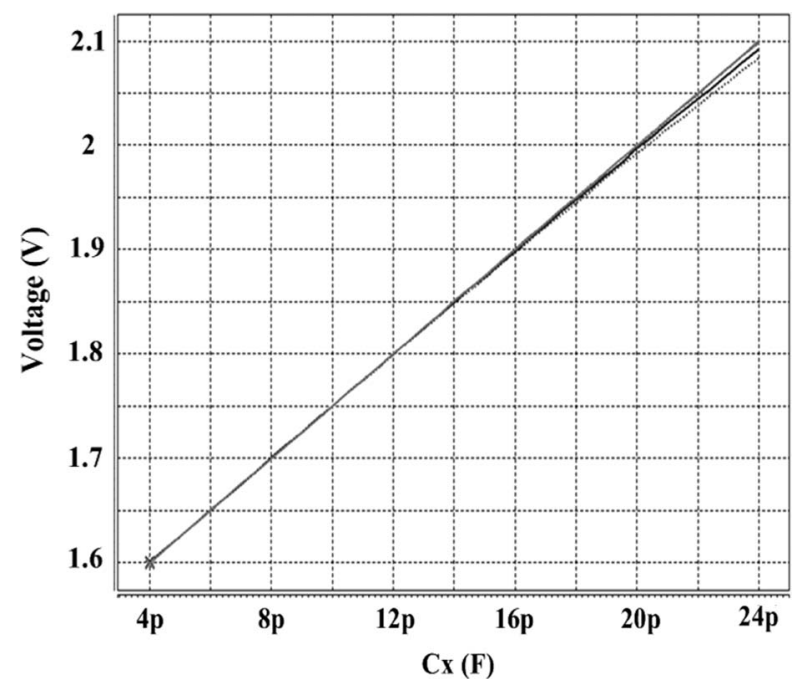

Fig. 10. SPICE simulation results of the CVC under the SPICE design corner (TT, SS, SF, FS, FF).

where the term

$$
\frac{\frac{\left(C_{F}+C_{R}\right)}{C_{F}}\left(V_{R 2}-V_{R 1}\right)}{A\left(\frac{C_{F}}{C_{F}+C_{R}+C_{x}}\right)}
$$

is the voltage error $\Delta V_{o}$, and $C_{F} /\left(C_{F}+C_{R}+C_{x}\right)$ is the feedback factor $\beta$. In the specification, the resolution of the CVC is designed to be 10 bits. Therefore, the voltage error $\Delta V_{o}$ could be given as follows:

$$
\frac{V_{i}}{2^{10}} \geq\left(\frac{C_{x}-C_{R}}{C_{F}}\right) \frac{\left(V_{R 2}-V_{R 1}\right)}{A \beta} .
$$




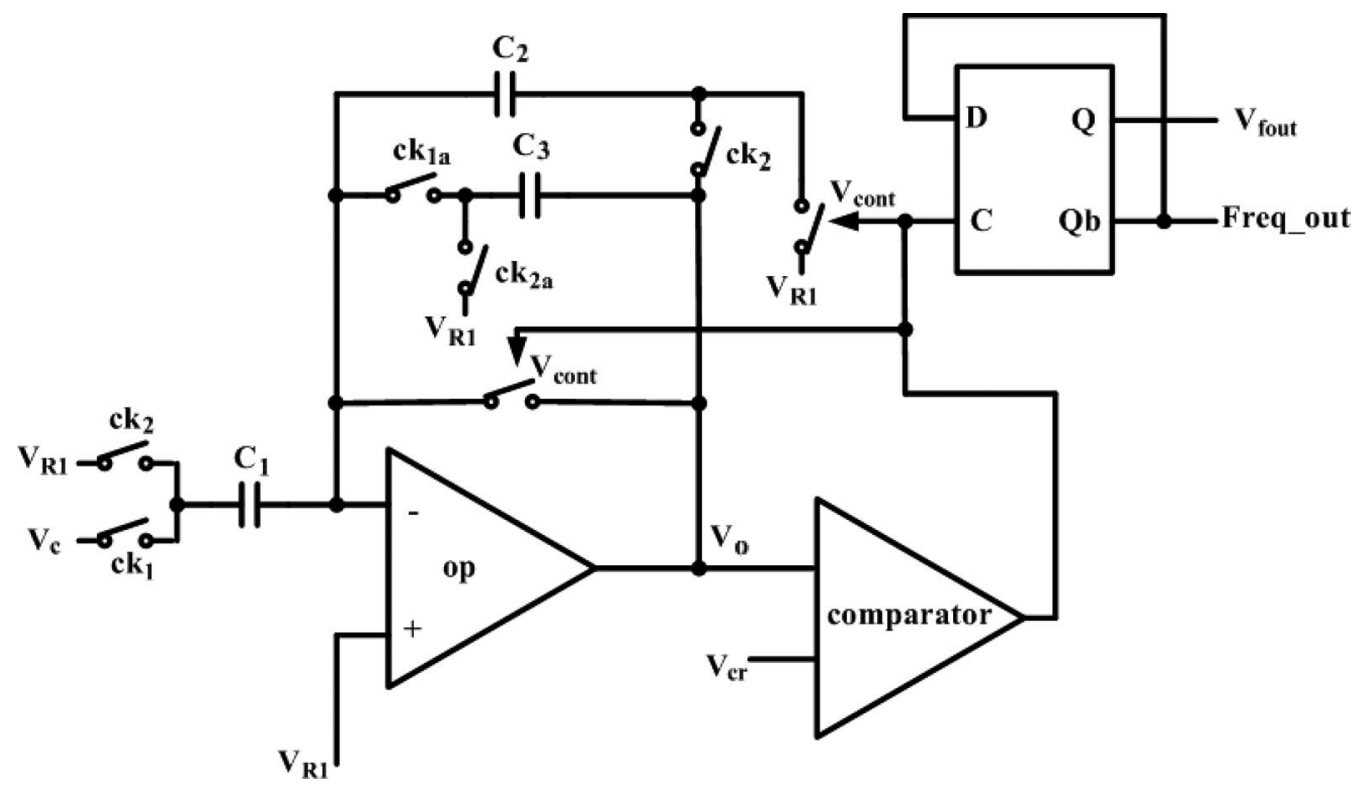

Fig. 11. Schematic of the VFC.

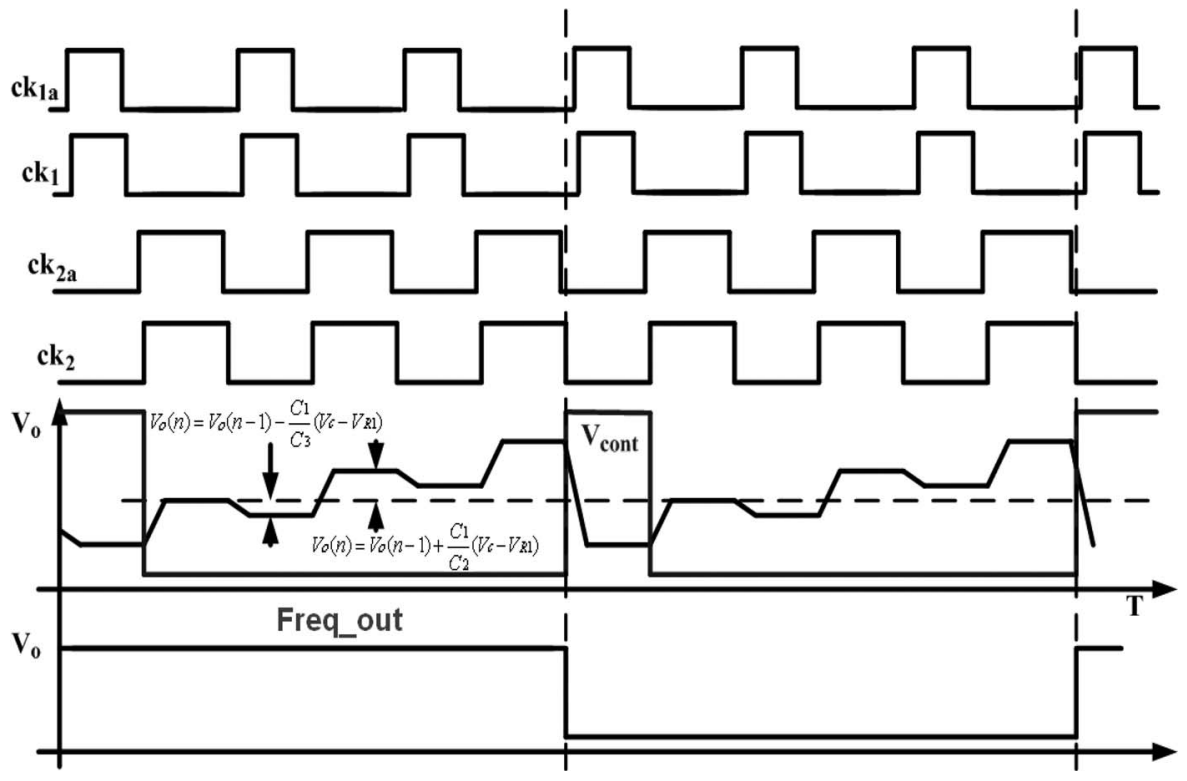

Fig. 12. Timing diagram of the VFC.

Thus, by performing (10), the OP gain should be higher than $67.82 \mathrm{~dB}$. Next, considering the bandwidth, the time constant is given by

$$
\tau=\frac{1}{\beta * W_{t}}=\frac{1}{2 \pi \beta * f_{t}} .
$$

To reserve the time for settling the circuit, the settling time $T_{\text {set }}$ is designed in the three-fourths clock period. Therefore, the settling time $T_{\text {set }}$ is given by

$$
T_{\mathrm{set}}=\frac{3}{4} \cdot \frac{1}{2} T_{c} \approx 180 \mathrm{~ns}
$$

where the system clock period $T_{c}$ is $500 \mathrm{~ns}$. The voltage error needs to be less than 1 least significant bit. Therefore

$$
\varepsilon=\left(V_{R 2}-V_{R 1}\right) \cdot e^{-T_{\mathrm{set}} / \tau} \leq \frac{V_{i}}{2^{10}} .
$$

Thus, by performing (13), the minimum unit-gain bandwidth $f_{t}$ is $15.3 \mathrm{MHz}$. Last, the consideration of the slew rate (SR) is given by

$$
\begin{aligned}
\mathrm{SR} & =\frac{I}{C_{L}}=\frac{V_{p-p}}{\frac{1}{4} * \frac{1}{2} * T_{c}} \\
V_{p-p} & =0.5 \mathrm{~V} .
\end{aligned}
$$

By performing (14), the SR should be larger than $8 \mathrm{~V} / \mu \mathrm{s}$. However, the parasitic capacitance of the detected capacitor $C_{x}$ 


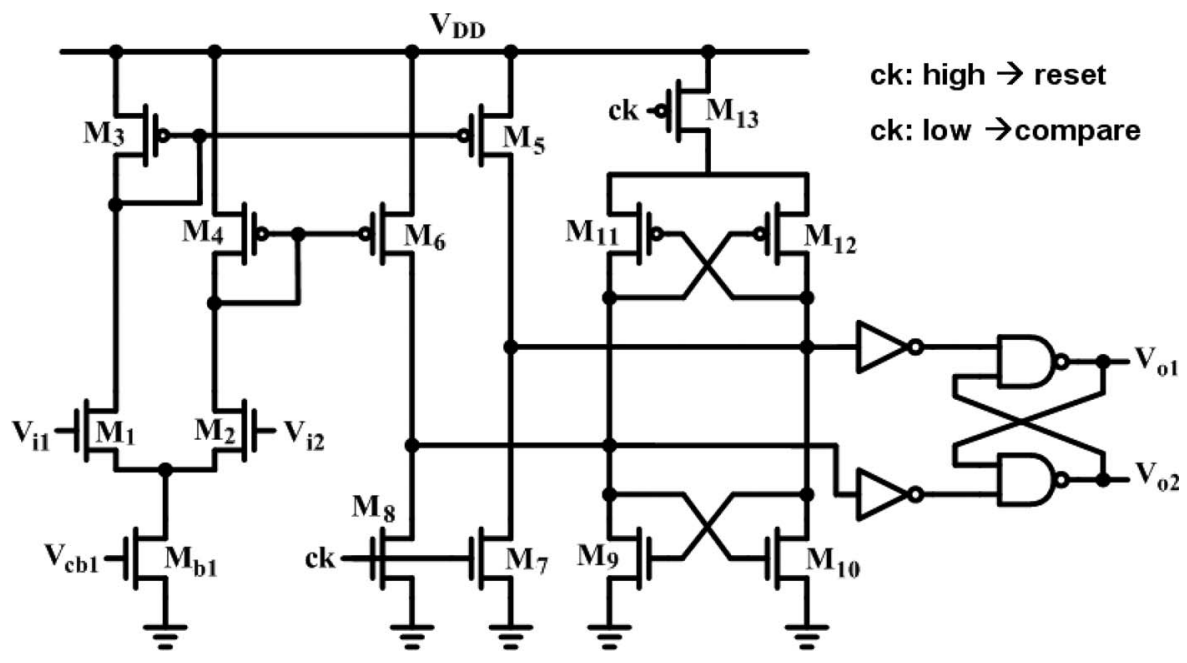

Fig. 13. Schematic of the comparator.

should be also considered. In Fig. 6, the parasitic capacitors $C_{s 1}, C_{s 2}$, and $C_{s 4}$ are connected to fixed biases; thus, they will not affect the charge transfer of the CVC. However, the parasitic capacitor $C_{s 3}$ in the negative input node of the OP will affect the charge transfer of the CVC. Let the parasitic capacitance of $C_{s 3}$ be equal to $5 \mathrm{pF}$. Therefore, (7) should be modified as follows:

$$
\begin{aligned}
& C_{x}\left(V_{R 2}-V_{R 1}\right)+C_{s 3} V_{R 1}=C_{x}\left(V_{R 1}-\mathrm{OP}_{-}\right) \\
& \quad+C_{R}\left(V_{R 2}-\mathrm{OP}_{-}\right)+C_{F}\left(V_{o}-\mathrm{OP}_{-}\right)+C_{s 3} \mathrm{OP}_{-} .
\end{aligned}
$$

Therefore

$$
V_{o}=\frac{\left(C_{x}-C_{R}\right)}{C_{F}}\left(V_{R 2}-V_{R 1}\right) *\left(1-\frac{1}{A\left(\frac{C_{F}}{C_{F}+C_{R}+C_{x}+C_{s 3}}\right)}\right) .
$$

By following (9)-(14) mentioned above, the OP gain should be $68.67 \mathrm{~dB}$, and the minimum unit-gain bandwidth $f_{t}$ is 16.9 MHz. The SR is the same, i.e., $8 \mathrm{~V} / \mu$ s. According to such specifications, the OP is designed and is shown in Fig. 7. The two-stage OP consists of the startup circuit $\left(M_{s 1}-M_{s 4}\right)$, the biasing circuit $\left(M_{11}-M_{16}\right)$, the first-stage amplifier $\left(M_{1}-M_{5}\right)$, the second-stage amplifier $\left(M_{6}\right.$ and $M_{7}$ ), and the compensation circuit ( $M_{10}$ used as a null resistor and a Miller compensation capacitor $C_{c}$ ). The $\mathrm{OP}$ under the frequency sweeping from $0.1 \mathrm{~Hz}$ to $1 \mathrm{GHz}$ is verified as shown in Fig. 8. The dc gain is $72 \mathrm{~dB}$, the unit gain bandwidth is $95 \mathrm{MHz}$, and the phase margin is $68^{\circ}$. The positive and negative SRs under the output loading $5 \mathrm{pF}$ are 248.5 and $41 \mathrm{~V} / \mu$ s, respectively. Fig. 9(a) and (b) presents the SPICE output waveforms under the capacitance $C_{x}$ of 24 and $12 \mathrm{pF}$, respectively. All the output voltages of the CVC circuit are plotted in Fig. 10. These results are simulated under the SPICE design corner (TT, SS, SF, FS, FF). All these results have rather linear relations between the capacitance and the voltage. These linear relations are obtained by considering the OP offset and switch charge injection insensitive properties. The SPICE simulation results and the characteristic of the CVC circuit are successfully verified and matched each other.
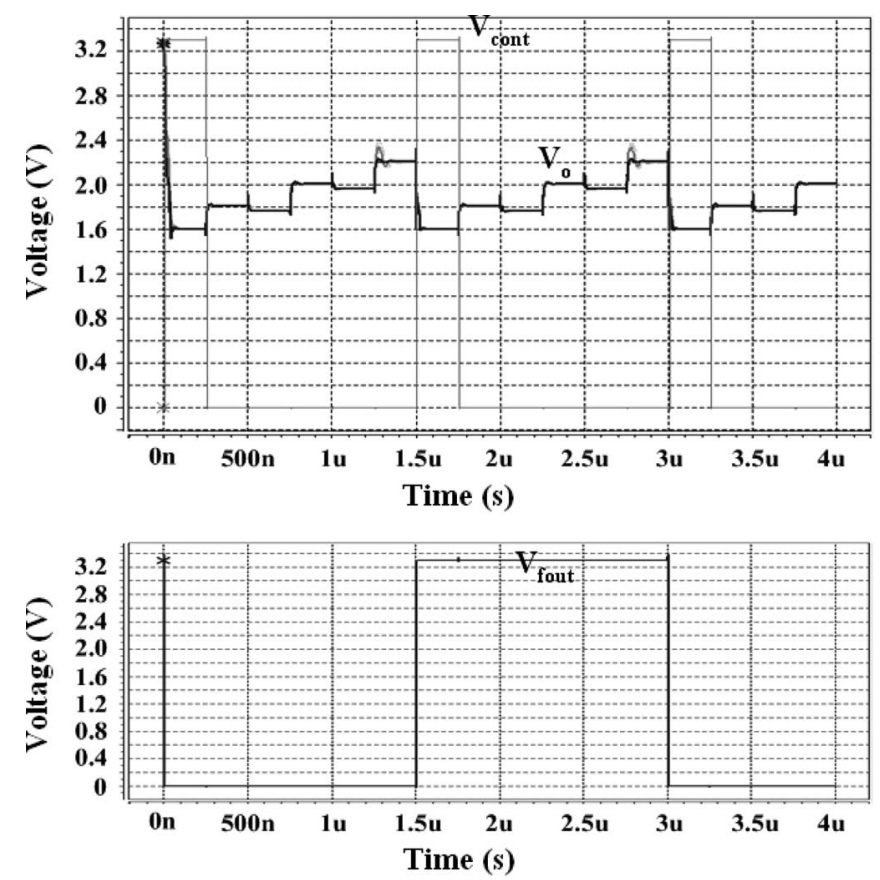

Fig. 14. SPICE output waveforms of the voltages $V_{\text {cont }}, V_{o}$, and $V_{\text {fout }}$ under the capacitance $C_{F}=20 \mathrm{pF}, C_{R}=4 \mathrm{pF}, C_{1}=1.5 \mathrm{pF}, C_{2}=3 \mathrm{pF}$, and $C_{3}=15 \mathrm{pF}$, the voltages $V_{R 1}=1.6 \mathrm{~V}$ and $V_{R 2}=2.1 \mathrm{~V}$, and the capacitance $C_{x}=20 \mathrm{pF}$.

\section{B. $V F C$}

The schematic of the VFC is shown in Fig. 11. It consists of the switch-capacitor integrator, a comparator, and a D flip-flop. Following the SC techniques, this circuit applies the charge redistribution method. These circuit operations could be written as follows:

$V_{o}(n)=V_{o}(n-1)+\frac{C_{1}}{C_{2}}\left(V_{c}-V_{R 1}\right)$ when the signal $c k_{2}$ is high

$V_{o}(n)=V_{o}(n-1)-\frac{C_{1}}{C_{3}}\left(V_{c}-V_{R 1}\right)$ when the signal $c k_{1}$ is high 


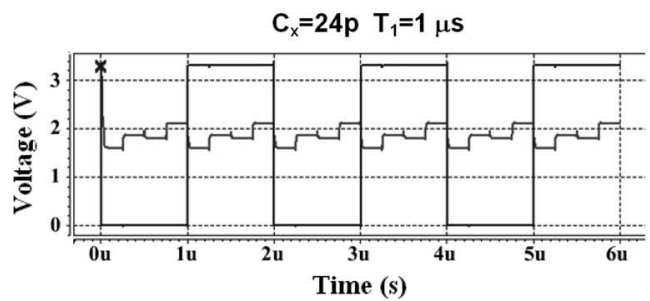

(a)

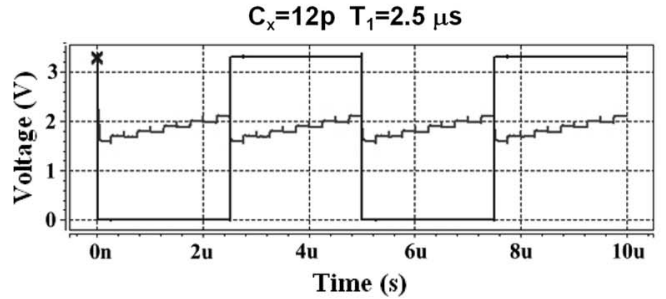

(c)

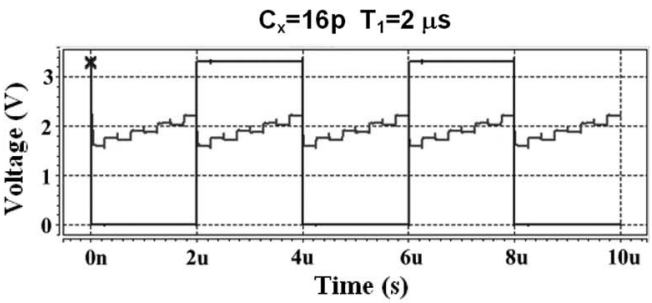

(b)

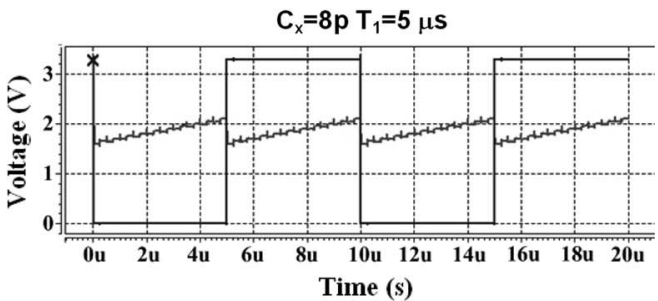

(d)

Fig. 15. SPICE output waveforms of the time period $T_{1}=1,2,2.5$, and $5 \mu$ s under the capacitance $C_{x}$ of (a) $24 \mathrm{pF}$, (b) $16 \mathrm{pF}$, (c) $12 \mathrm{pF}$, and (d) $8 \mathrm{pF}$.

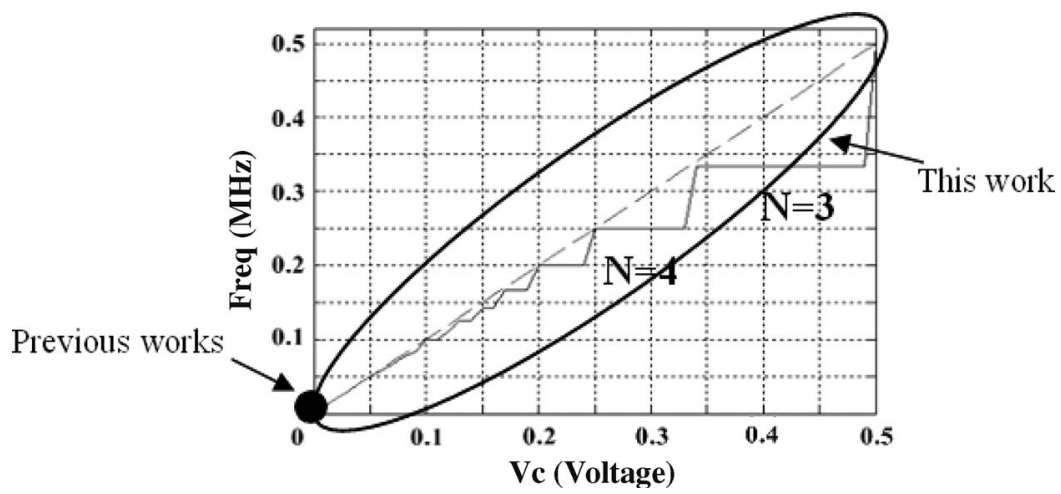

Fig. 16. Relation between the voltage and the frequency of the VFC (without automatic compensation circuits).

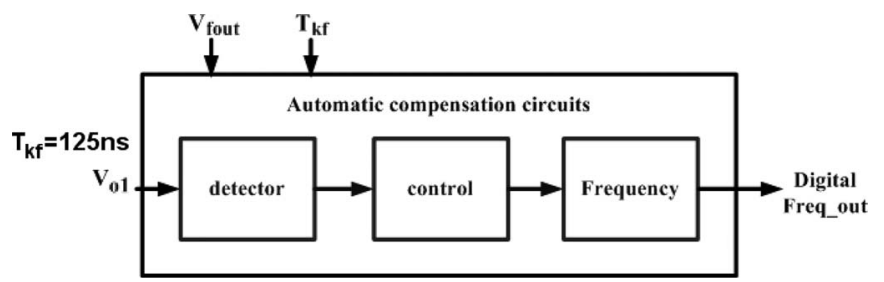

Fig. 17. Block diagram of the automatic compensation circuits.

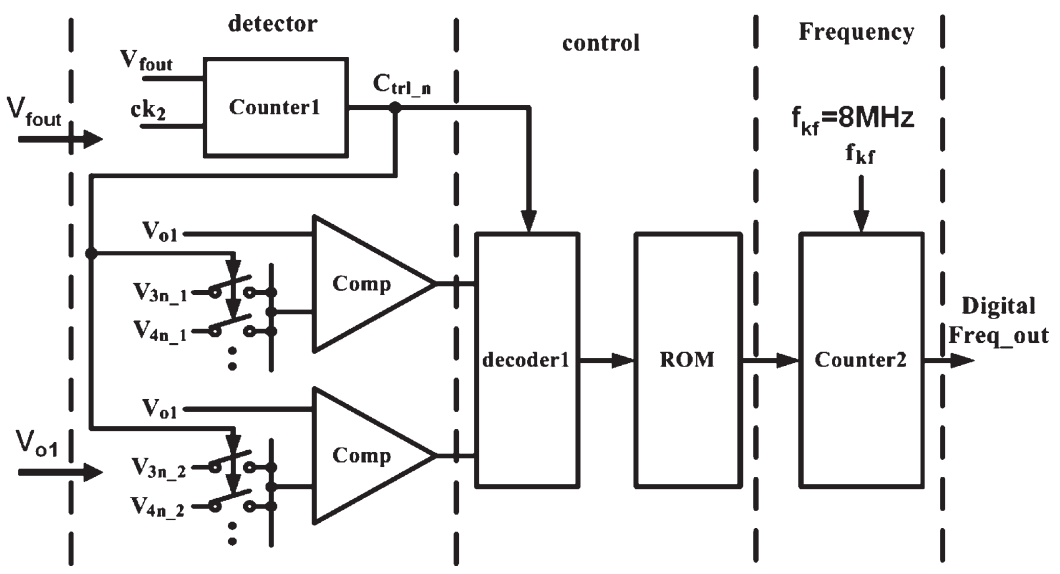

Fig. 18. Schematic of the automatic compensation circuits. 


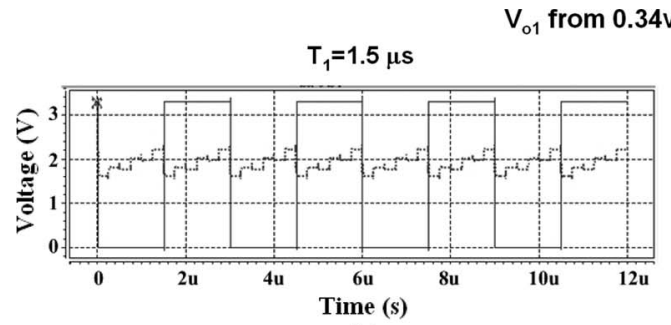

(a)

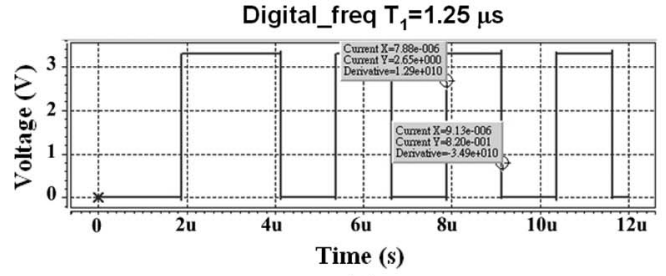

(c)
Digital_freq $T_{1}=1.125 \mu \mathrm{s}$

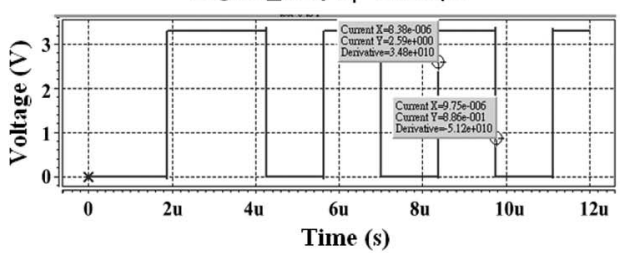

(b)

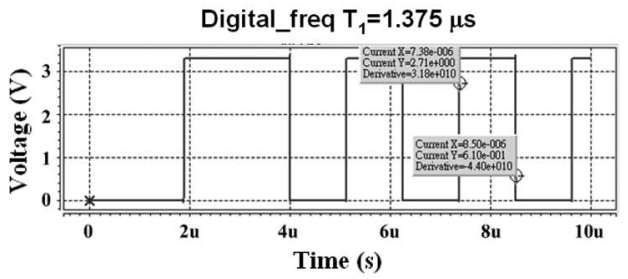

(d)

Fig. 19. SPICE output waveforms of the automatic compensation circuits under step number $N=3$. The time periods $T_{1}$ are (a) $1.5 \mu$ s, (b) $1.125 \mu$ s, (c) $1.25 \mu \mathrm{s}$, and (d) $1.375 \mu \mathrm{s}$.

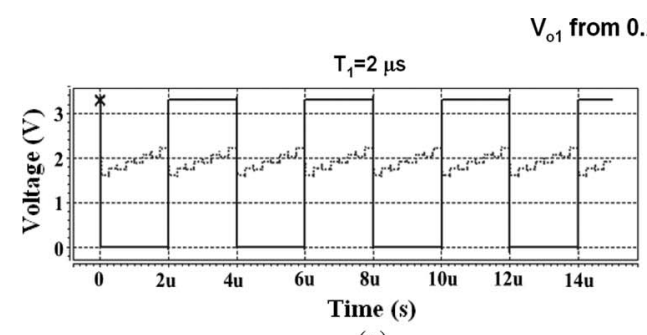

(a)

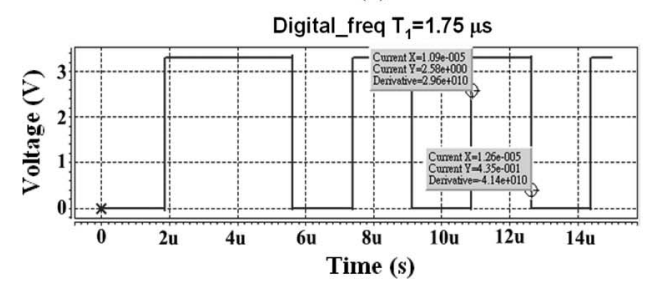

(c)

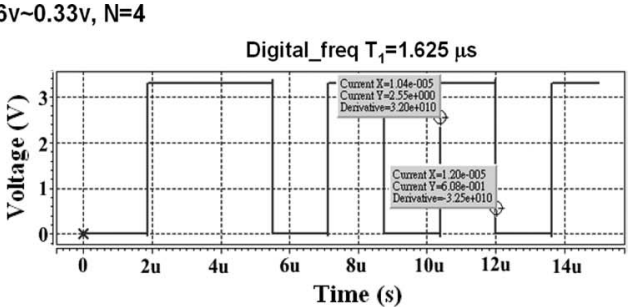

(b)

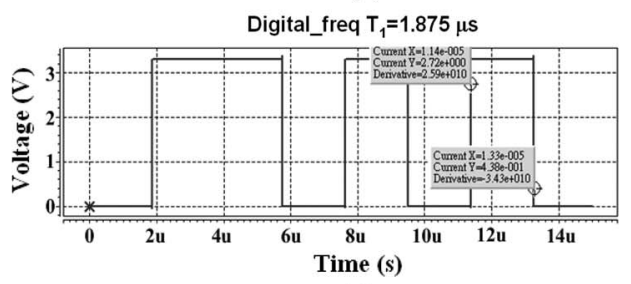

(d)

Fig. 20. SPICE output waveforms of the automatic compensation circuits under step number $N=4$. The time periods $T_{1}$ are (a) $2 \mu$ s, (b) $1.625 \mu$ s, (c) $1.75 \mu \mathrm{s}$, and (d) $1.875 \mu \mathrm{s}$.

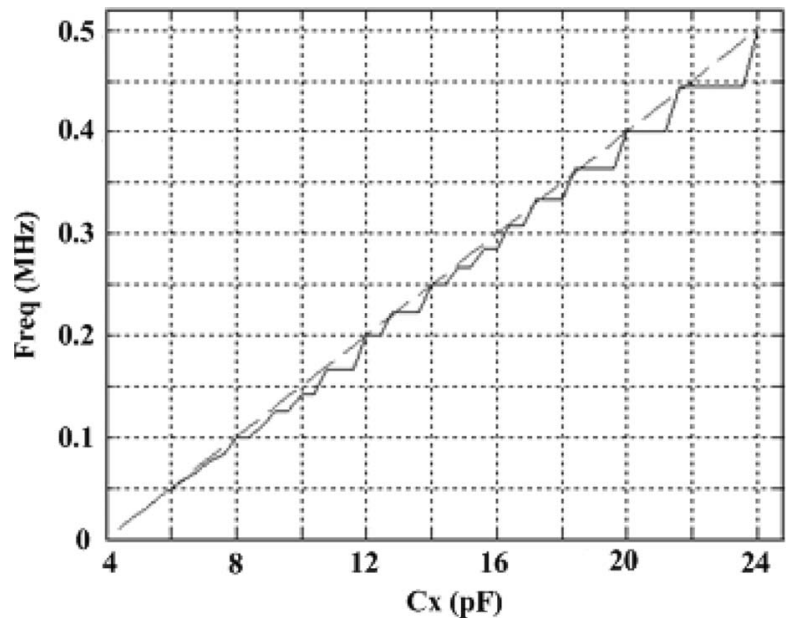

Fig. 21. Relation between the capacitance and the frequency of the proposed autocompensated sensor transducer.
TABLE II

DeVice Parameters Used on the Proposed AUTOCOMPENSATED SENSOR TRANSDUCER

\begin{tabular}{|c|c|c|c|c|c|c|c|c|c|}
\hline $\mathrm{C}_{\mathrm{x}}$ & $\mathrm{C}_{\mathrm{R}}$ & $\mathrm{C}_{\mathrm{F}}$ & $\mathrm{C}_{1}$ & $\mathrm{C}_{2}$ & $\mathrm{C}_{3}$ & $\mathrm{f}_{\mathrm{c}}$ & $\mathrm{f}_{\mathrm{kf}}$ & $\mathrm{V}_{\mathrm{cr}}$ & $\mathrm{F}$ \\
\hline $4 \sim 24 \mathrm{p}$ & $4 \mathrm{p}$ & $20 \mathrm{p}$ & $1.5 \mathrm{p}$ & $3 \mathrm{p}$ & $15 \mathrm{p}$ & $2 \mathrm{MHz}$ & $8 \mathrm{MHz}$ & $2.1 \mathrm{~V}$ & $0.5 \sim 500 \mathrm{kHz}$ \\
\hline
\end{tabular}

where $n$ is defined as the $n$th cycle of the switch-capacitor integrator, and $V_{c}$ is the output voltage of the CVC circuit. The capacitor ratio $C_{1} / C_{2}$ is five to ten times the ratio of $C_{1} / C_{3}$. Therefore, the voltage $V_{o}$ can step up until it exceeds the threshold voltage $V_{c r}$ of the comparator. When the voltage $V_{o}$ is higher than the voltage $V_{c r}$, the voltage $V_{c o n t}$ will go to logic high and reset the voltage $V_{o}$. The D flip-flop is also triggered. Moreover, when the voltage $V_{\text {cont }}$ is logic high, the capacitor $C_{2}$ will store the $\mathrm{OP}$ offset voltage and perform the circuit operation of the offset cancellation. All the timing 


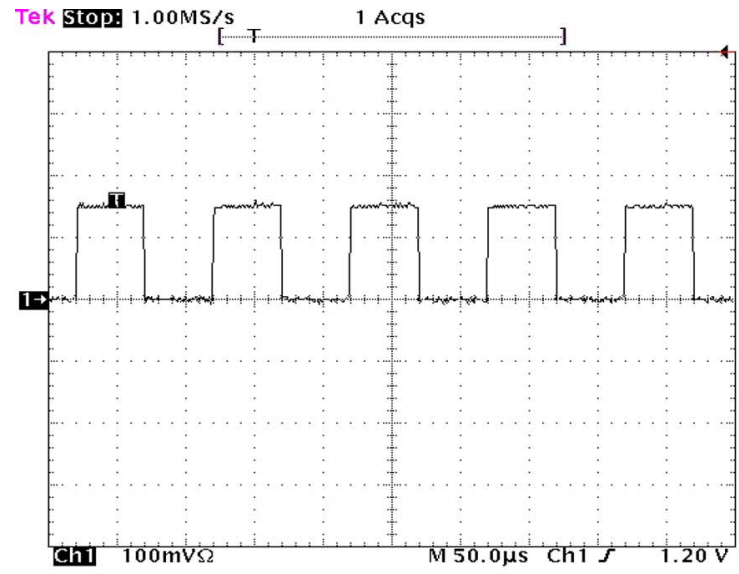

(a)

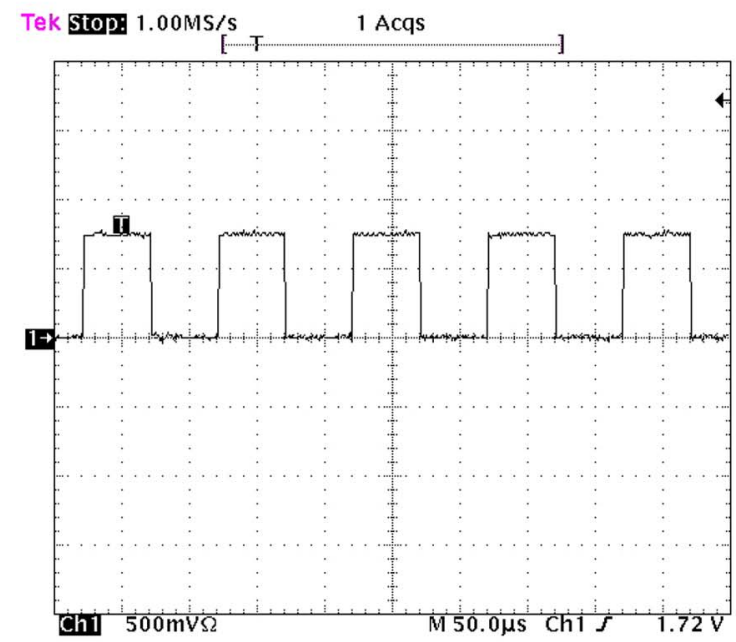

(c)

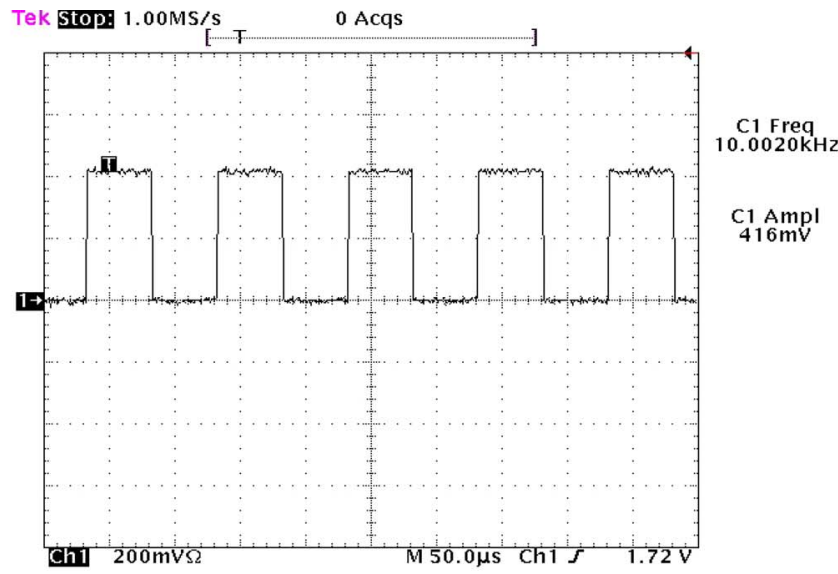

(b)

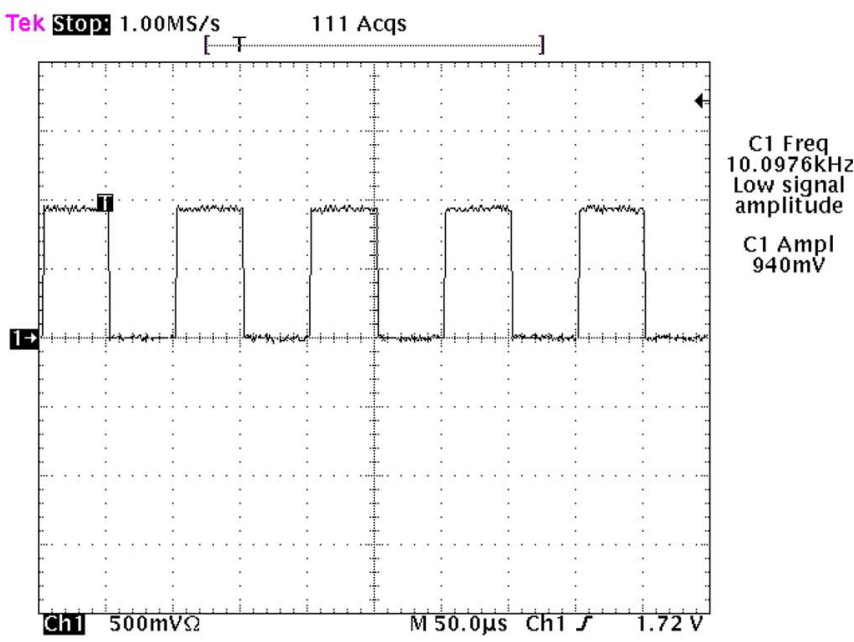

(d)

Fig. 22. Measured results of the CVC under the capacitance $C_{x}$ of (a) $6.8 \mathrm{pF}$, (b) $12 \mathrm{pF}$, (c) $18 \mathrm{pF}$, and (d) $22 \mathrm{pF}$.

signals discussed above are plotted in Fig. 12. The output frequency $F$ of the VFC circuit is derived as

$$
\begin{aligned}
F & =\frac{1}{T}=\frac{1}{2 T_{1}}=\frac{1}{2 * N * T_{c}}=\frac{1}{2 *\left(\frac{V_{c r}-V_{R 1}}{V_{c}-V_{R 1}} * \frac{C_{2}}{C_{1}}\right) * T_{c}} \\
& =\frac{1}{2} * \frac{C_{1}}{C_{2}} \frac{V_{c}-V_{R 1}}{V_{c r}-V_{R 1}} f_{c}
\end{aligned}
$$

where $N$ is the step number, and $f_{c}$ is the reciprocal of the system clock period $T_{c}$. Hence, combining (5) and (19), the output frequency $F$ could be modified as

$$
F=\frac{1}{2} * \frac{C_{1}}{C_{2}} * \frac{C_{x}-C_{R}}{C_{F}} * \frac{V_{R 2}-V_{R 1}}{V_{c r}-V_{R 1}} f_{c} .
$$

Fig. 13 shows the schematic of the comparator circuit. When the signal $c k$ goes to logic low, the comparison of the two input differential pairs is performed. The SPICE output waveforms of the voltages $V_{\text {cont }}, V_{o}$, and $V_{\text {fout }}$ are presented in Fig. 14. Last, the output frequency of the VFC circuit is simulated in Fig. 15(a)-(d). In Fig. 15(b), by performing (20), the output time period $T_{1}$ should be $5 / 3 \mu \mathrm{s}$. However, it does not match the simulation result of $2 \mu \mathrm{s}$. Moreover, the nonlinearity on the high-frequency range is shown in Fig. 16. The reason for this nonlinearity is that the step number $N$ must be an integer

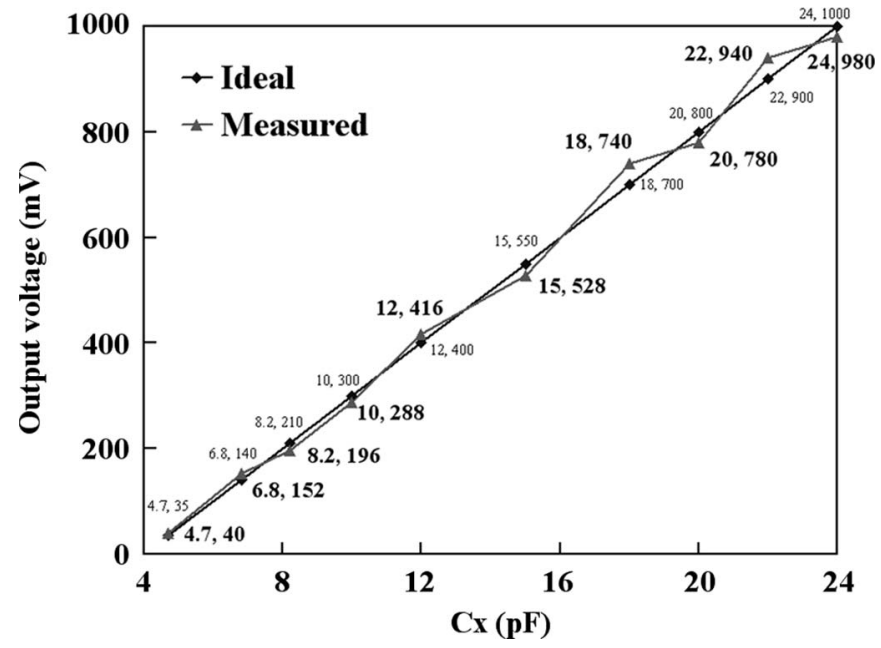

Fig. 23. Measured results of the $\mathrm{CVC}$ under the varied capacitance of $C_{x}$, which ranges from 4.7 to $24 \mathrm{pF}$. The boldface-type numbers are the measured values, and the fine-type numbers are the ideal values.

number. Hence, this effect will produce the quantized error. The previous works [5]-[8] do not meet this problem because their frequency band is lower. To solve this problem of obtaining the wide dynamic frequency range, the digitized compensation circuits are, thus, newly investigated [9]. 


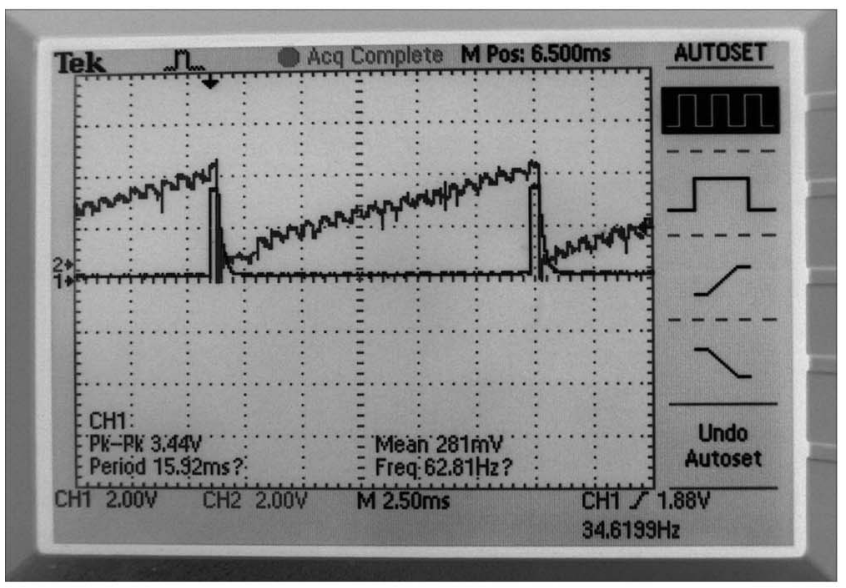

(a)

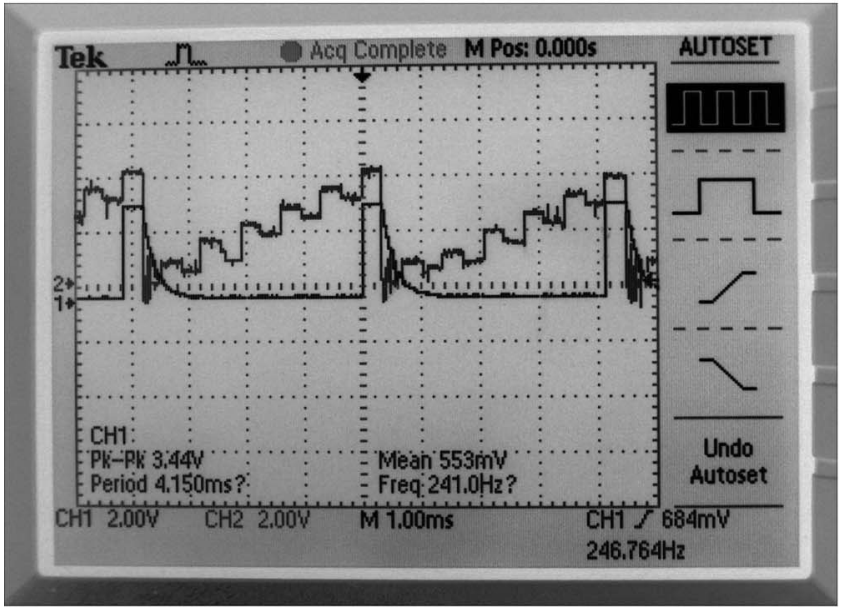

(c)

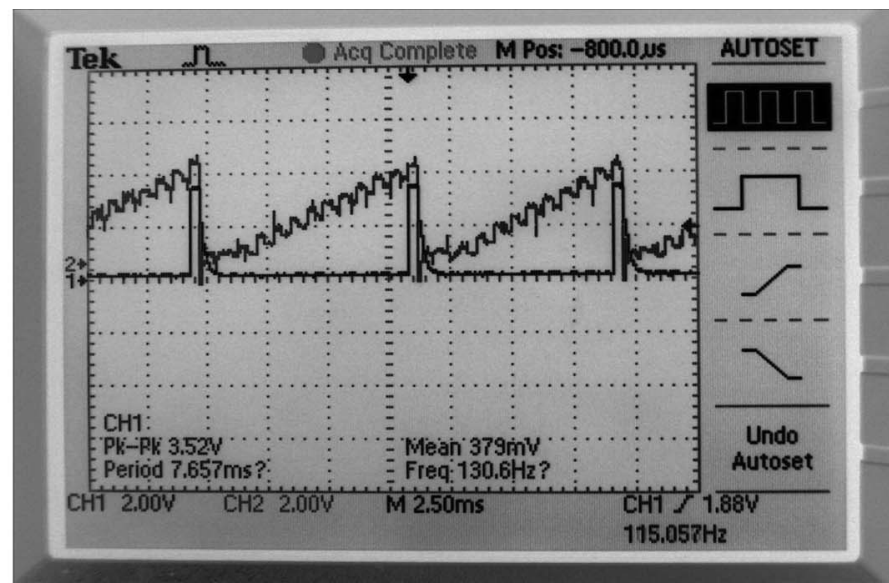

(b)

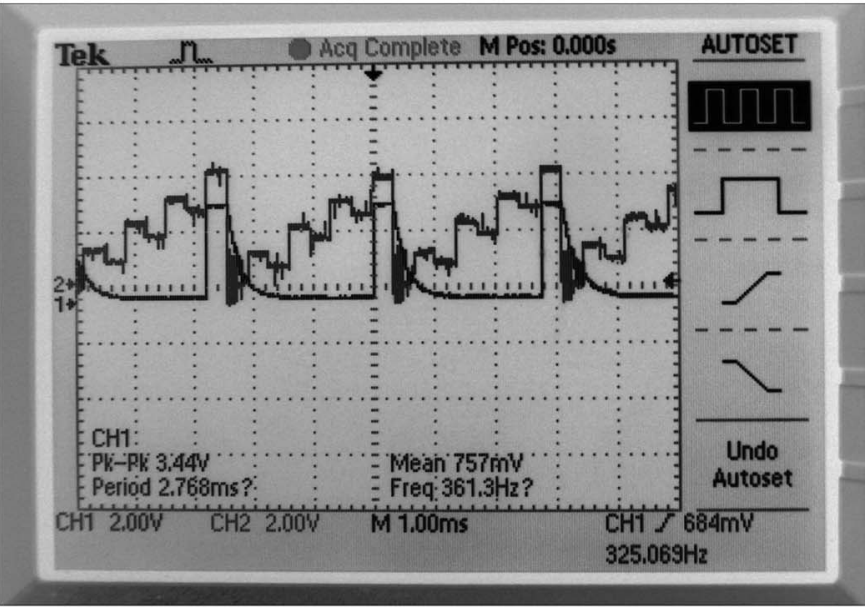

(d)

Fig. 24. Measured results of the VFC under the voltage $V_{c}$ of (a) $0.44 \mathrm{~V}$, (b) $0.74 \mathrm{~V}$, (c) $1.35 \mathrm{~V}$, and (d) $2 \mathrm{~V}$.

\section{Automatic Compensation Circuits}

In Fig. 17, the nonlinearity is obviously observed on $N=$ $3\left(0.34<V_{c}<0.49 \mathrm{~V}\right)$ and $N=4\left(0.25<V_{c}<0.34 \mathrm{~V}\right)$. Therefore, to obtain a wide dynamic frequency range, the automatic compensation circuits are proposed to compensate the nonlinearity of the VFC on the range of step numbers 3 and 4. The design concept of the automatic compensation circuits is the performance of the interpolation method. In the circuit implementation, the automatic compensation circuits include the detector, control, and frequency circuits, as shown in Fig. 18. Depending on the two voltages $V_{\text {font }}$ and $V_{o 1}$, which are the output signals of the VFC and CVC circuits, the detector will decide the step number $N$. Then, the control circuit will perform the compensation algorithm and send the decision signals to the frequency circuit. Last, the frequency circuit generates the desired compensation frequency and outputs the frequency signal through the digital buffer. The compensation algorithm is performed as follows, where $T_{k f}$ is the sampling period (125 ns), and $V_{\text {ref } 1}$ and $V_{\text {ref } 2}$ are the threshold voltages of the comparators. Now, the circuit operations to perform the compensation algorithm are described in Fig. 18. First, in the detector block, counter 1 counts and determines the step number $N$. Then, the control signal $C_{t r l \_n}$ will be used to turn on the

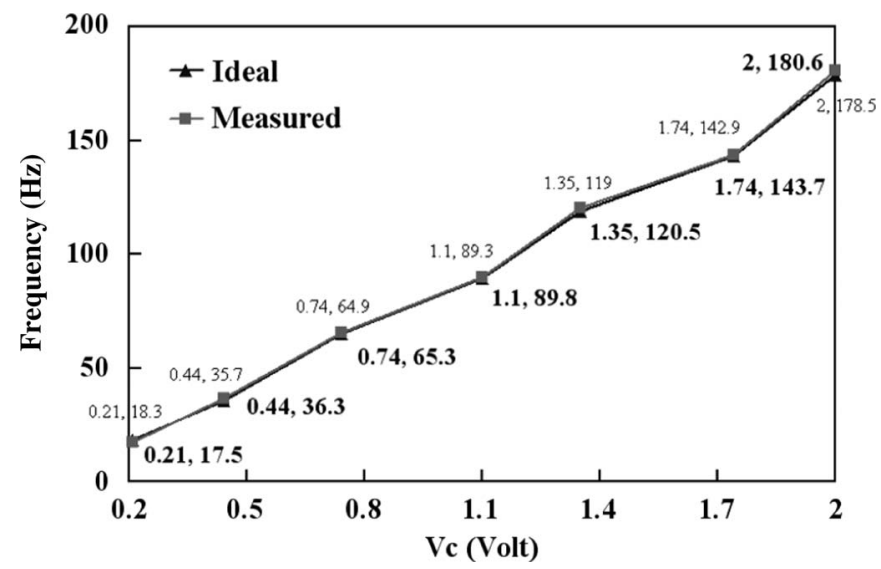

Fig. 25. Measured results of the VFC under the varied voltage $V_{c}$, which ranges from 0.21 to $2 \mathrm{~V}$. The boldface-type numbers are the measured values, and the fine-type numbers are the ideal values.

switches, and comparators will use the reference voltages $V_{3 n \_1}$ and $V_{3 n \_2}$, which belonged to $N=3$, or the reference voltages $V_{4 n \_1}$ and $V_{4 n \_2}$, which belonged to $N=4$. The outputs of the comparators are thermometer codes. By using these codes, decoder1 can grab the stored data of the ROM in the control block. The stored data in the ROM, which are numbers 9-15, 


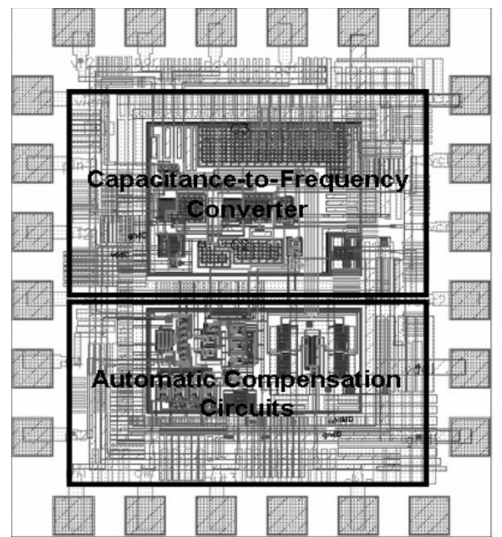

(a)

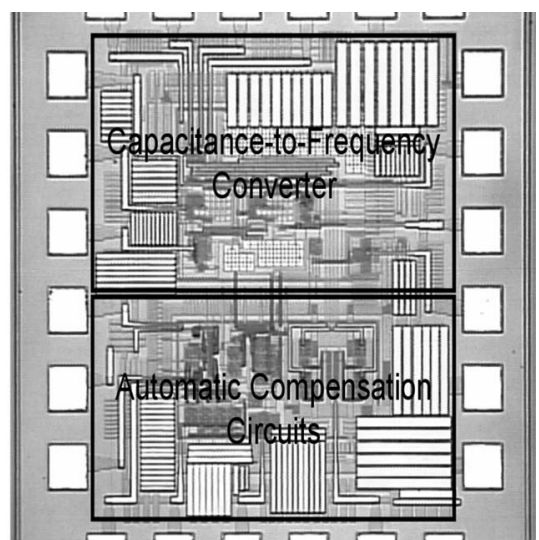

(b)

Fig. 26. (a) Physical layout and (b) photograph of the fabricated autocompensated sensor transducer. The area of this implemented chip is $940 \times 1080 \mu \mathrm{m}^{2}$.

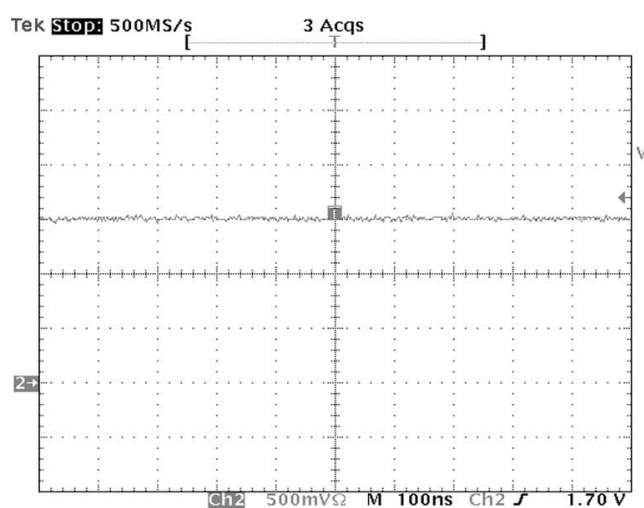

(a)

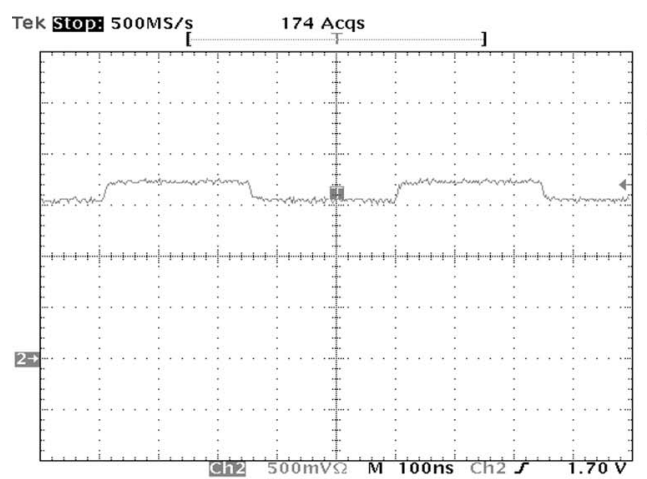

(c)

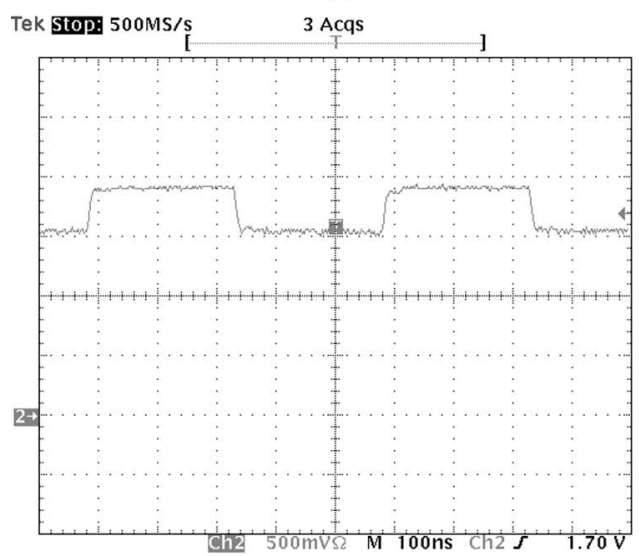

(e)

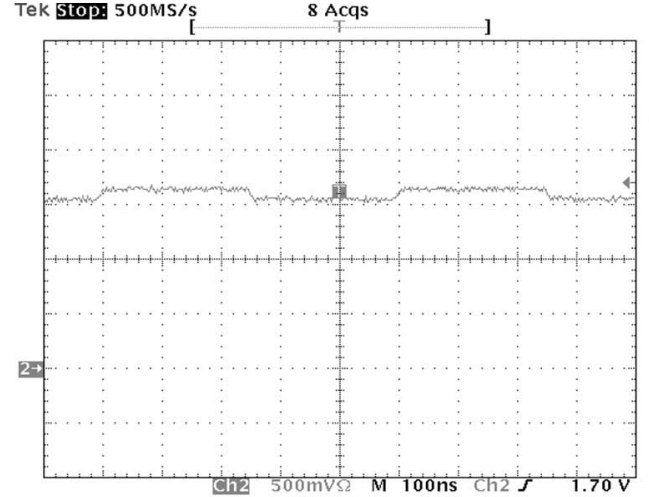

(b)

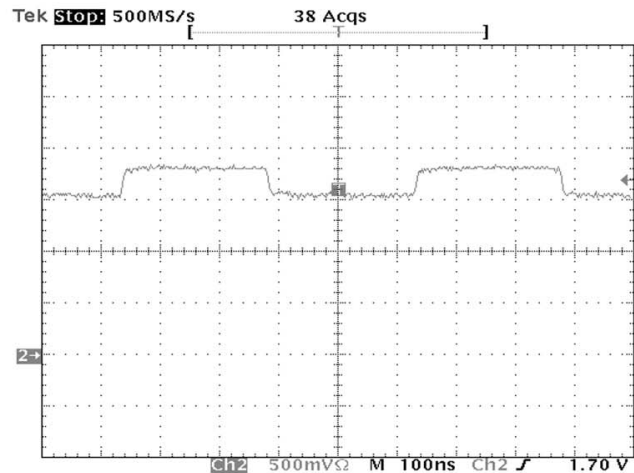

(d)

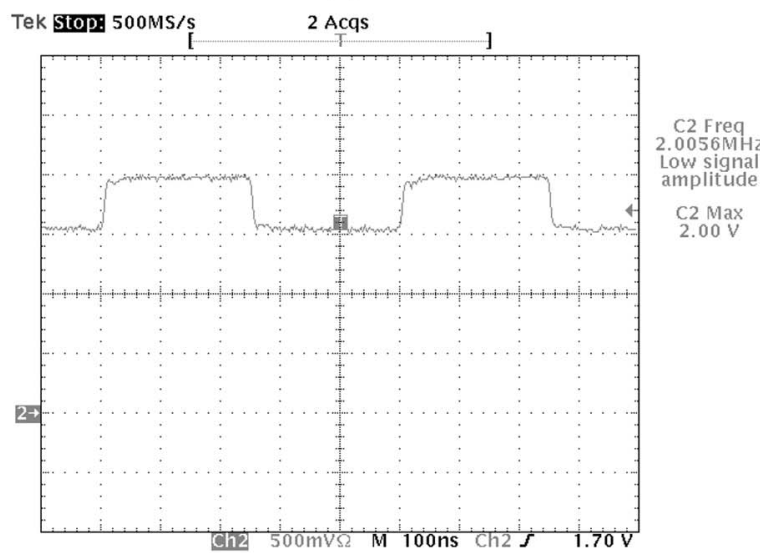

(f)

Fig. 27. Measured results of the integrated CVC under the capacitance $C_{x}$ of (a) $4 \mathrm{pF}$, (b) $8 \mathrm{pF}$, (c) $12 \mathrm{pF}$, (d) $16 \mathrm{pF}$, (e) $20 \mathrm{pF}$, and (f) $22 \mathrm{pF}$. 
will be sent to the next block. Last, counter2 will generate the compensated frequency. For example, if step number $N$ is determined to be 3 and the chosen number from the ROM is 10 , the output compensated time period $T_{1}$ will be $1250 \mathrm{~ns}$. Thus, by performing the automatic compensation algorithm, the nonlinearity of the VFC on the range of step numbers 3 and 4 can be compensated. Last, the wide dynamic frequency range is obtained. Figs. 19 and 20 demonstrate the SPICE output waveforms of the automatic compensation circuits under step numbers 3 and 4, respectively. Fig. 21 shows the linear relation between the capacitance and the frequency of the autocompensated sensor transducer. Obviously, the quantized error has been decreased, and the automatic compensation circuits successfully function as well. Simulation results above have confirmed the correct functions and performance of the proposed autocompensated sensor transducer. All the device parameters that are used on the proposed autocompensated sensor transducer are listed in Table II.

\section{When $N=3$}

if the voltage $V_{o 1}>V_{r e f 1}$, then the compensated time period $T_{1}=9 * T_{k f}$;

If the voltage $V_{r e f 1}>V_{o 1}>V_{\text {ref } 2}$, then the compensated time period $T_{1}=10 * T_{k f}$

if the voltage $V_{\text {ref2 } 2}>V_{o 1}$, then the compensated time period $T_{1}=11 * T_{k f}$.

\section{When $N=4$}

if the voltage $V_{o 1}>V_{r e f 1}$, then the compensated time period $T_{1}=13 * T_{k f}$;

if the voltage $V_{\text {ref } 1}>V_{o 1}>V_{\text {ref } 2}$, then the compensated time period $T_{1}=14 * T_{k f}$;

if the voltage $V_{\text {ref } 2}>V_{o 1}$, then the compensated time period $T_{1}=15 * T_{k f}$.

\section{Measurement Results}

First, the circuits of the CVC and the VFC are built by discrete components to verify circuit operations. The switches, an $\mathrm{OP}$, and a comparator are implemented with analog bilateral switches CD4066, LF411, and LM311, respectively. The capacitance values of $C_{R}, C_{F}, C_{1}, C_{2}$, and $C_{3}$ are $4,40,5$, 10 , and $45 \mathrm{pF}$, respectively. The system clock frequency $f_{c}$ is $1.45 \mathrm{kHz}$, and the reference voltages $V_{R 1}, V_{R 2}$, and $V_{c r}$ are 0,2 , and $4 \mathrm{~V}$, respectively. First, by performing the circuit operation of the CVC, the measured results are demonstrated in Fig. 22 under the capacitance $C_{x}$ of $6.8,12,18$, and $22 \mathrm{pF}$ [for Fig. 22(a)-(d), respectively]. The output voltages are 152, 416, 740, and $940 \mathrm{mV}$, respectively. All the results are plotted in Fig. 23, and the accuracy is within $\pm 8.57 \%$. Fig. 24 shows the measured results of the VFC under the voltage $V_{c}$ of 0.44 , 0.74, 1.35, and $2 \mathrm{~V}$ [for Fig. 24(a)-(d), respectively]. Taking Fig. 24(a) as an analysis example, when the voltage $V_{c}$ is $0.44 \mathrm{~V}$, the step-up voltage is $0.22 \mathrm{~V}$. The voltage $V_{o}$, as shown in Fig. 12, will continue to go up until it exceeds the reference voltage $V_{c r}$ of the comparator. The step number $N$ is 20 . The time period $T_{1}$ is equal to $14 \mathrm{~ms}(20 * 0.7 \mathrm{~ms})$. To verify the time period $T_{1}$ in Fig. 24(a), each timescale is $2.5 \mathrm{~ms}$, and

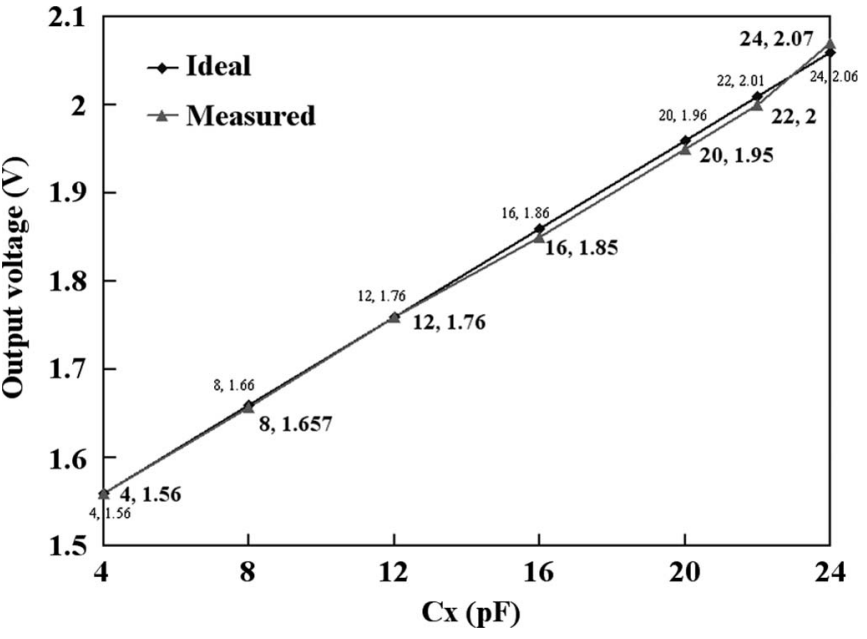

Fig. 28. Measured results of the integrated CVC under the varied capacitance of $C_{x}$, which ranges from 4 to $24 \mathrm{pF}$. The boldface-type numbers are the measured values, and the fine-type numbers are the ideal values.

the time period between two $V_{\text {cont }}$ signals is around five to six timescales. Thus, the time period is about $13.75 \mathrm{~ms}$. This measured result matched the calculation result of $14 \mathrm{~ms}$. By the same way, in Fig. 24(b), the voltage $V_{c}$ is $0.74 \mathrm{~V}$, and the step-up voltage is $0.37 \mathrm{~V}$. The step number $N$ is 11 . The time period $T_{1}$ is equal to $7.7 \mathrm{~ms}(11 * 0.7 \mathrm{~ms})$, which matched the measured result of $7.657 \mathrm{~ms}$. The time periods $T_{1}$ of Fig. 21(c) and (d) are $4.2(6 * 0.7)$ and $2.8(4 * 0.7) \mathrm{ms}$, respectively. They are also successfully proven to match the measured results, which are 4.15 and $2.768 \mathrm{~ms}$. All the results are plotted in Fig. 25, and the accuracy is within $\pm 4.37 \%$. Thus, all the circuit operations of the CVC and the VFC are successfully verified.

Last, the monolithic CMOS autocompensated sensor transducer for capacitive measuring systems has been implemented. Fig. 26(a) and (b) demonstrates the physical layout and the photograph of the fabricated autocompensated sensor transducer, respectively. The area of this implemented chip is $940 \times$ $1080 \mu \mathrm{m}^{2}$, and the power consumption is $6.4 \mathrm{~mW}$. The system clock frequency $f_{c}$ is $2 \mathrm{MHz}$, and the reference voltages $V_{R 1}$, $V_{R 2}$, and $V_{c r}$ are $1.6,2.1$, and $2.1 \mathrm{~V}$, respectively. First, by performing the circuit operation of the integrated CVC, the measured results under the capacitance $C_{x}$ of $4,8,12,16$, 20, and $22 \mathrm{pF}$ are demonstrated in Fig. 27(a)-(f), respectively. All the results are plotted in Fig. 28, and the accuracy is within $\pm 3.33 \%$. Fig. 29 shows the measured results of the integrated CFC under the capacitance $C_{x}$ of $6.6,8,12,16,20$, 22, and $24 \mathrm{pF}$ [for Fig. 29(a)-(g), respectively]. As discussed in Section III, the nonlinearity produced by the quantized error on the high-frequency range is also measured and is shown in Fig. 30. However, after performing the automatic compensation circuits, the nonlinearity has been greatly reduced, and the compensated output frequency is shown in Fig. 29(h)-(j). The accuracy is improved and promoted from $\pm 25.77 \%$ (without compensation) to $\pm 5.14 \%$. Thus, as in Section III, the automatic compensation circuits enhance and compensate the linear relation between the variable capacitance of the detected sensor and the output digital frequency. Last, Fig. 31(a) and (b) displays the measurement setup and the measured results of 


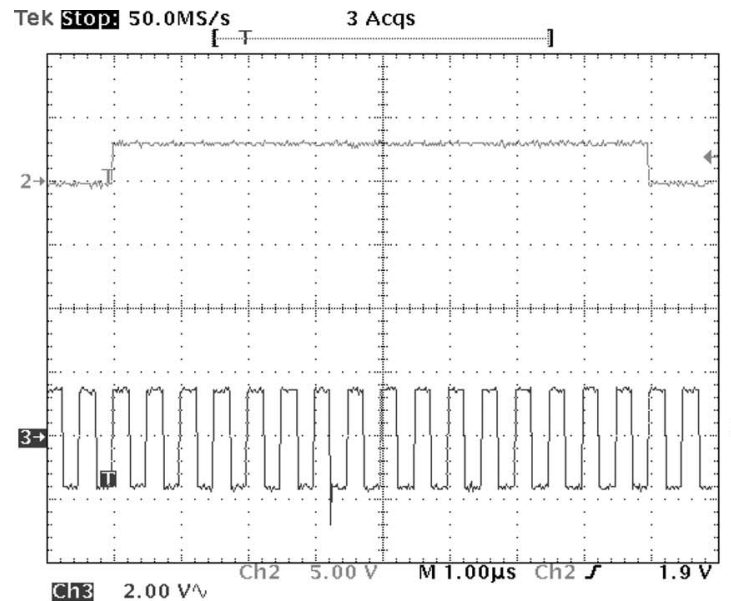

(a)

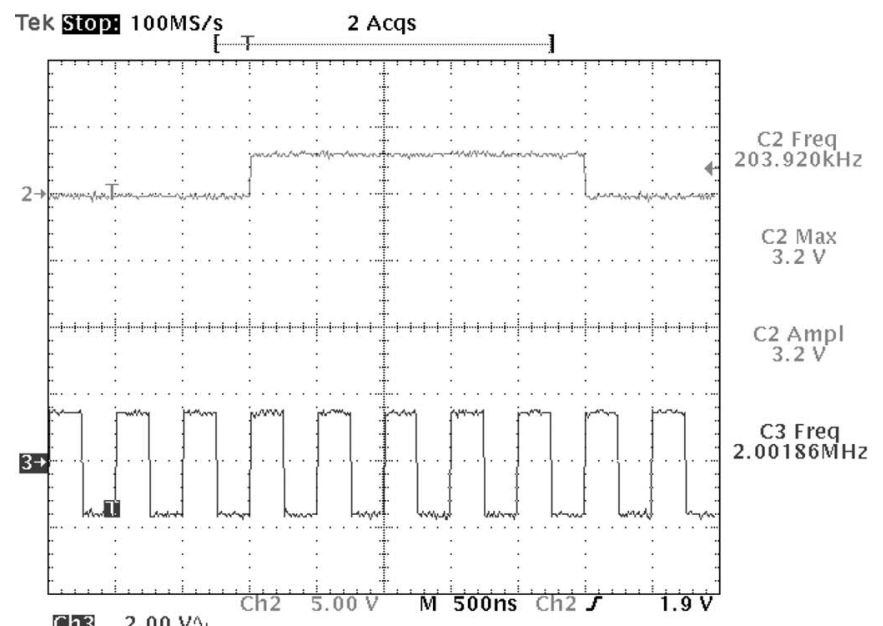

(c)

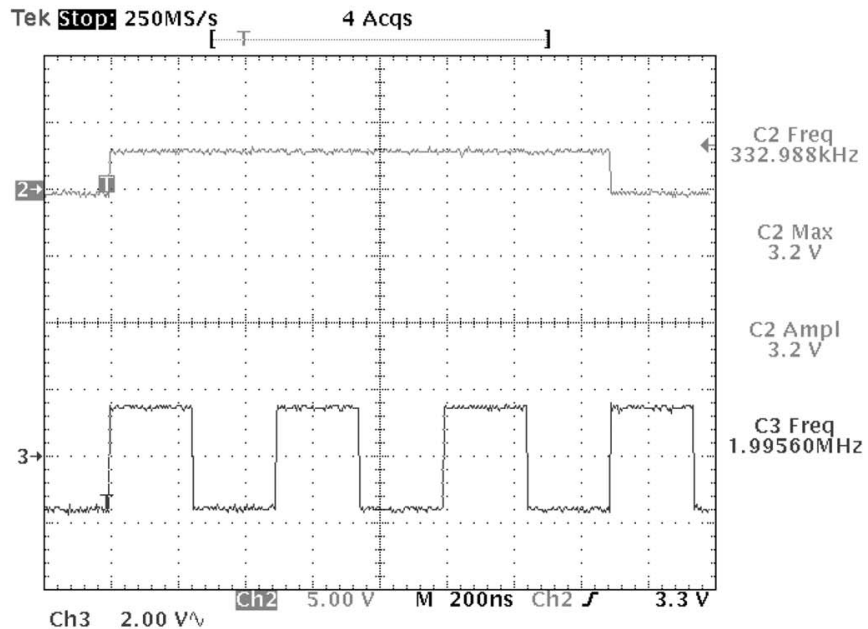

(e)

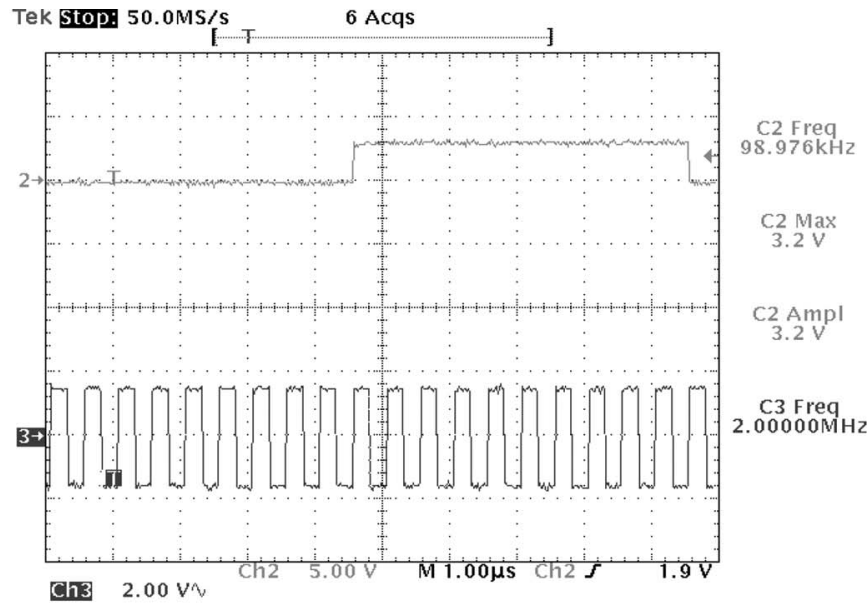

(b)

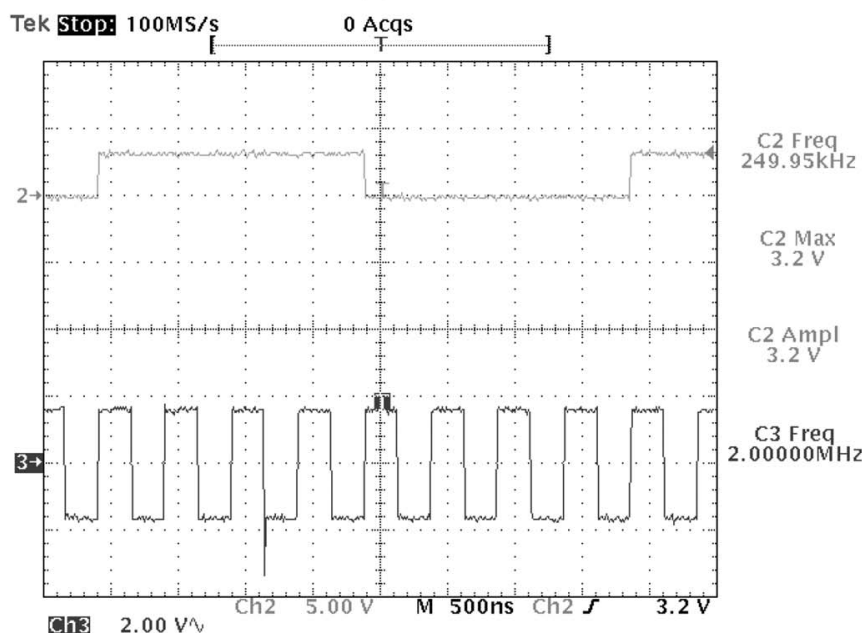

(d)

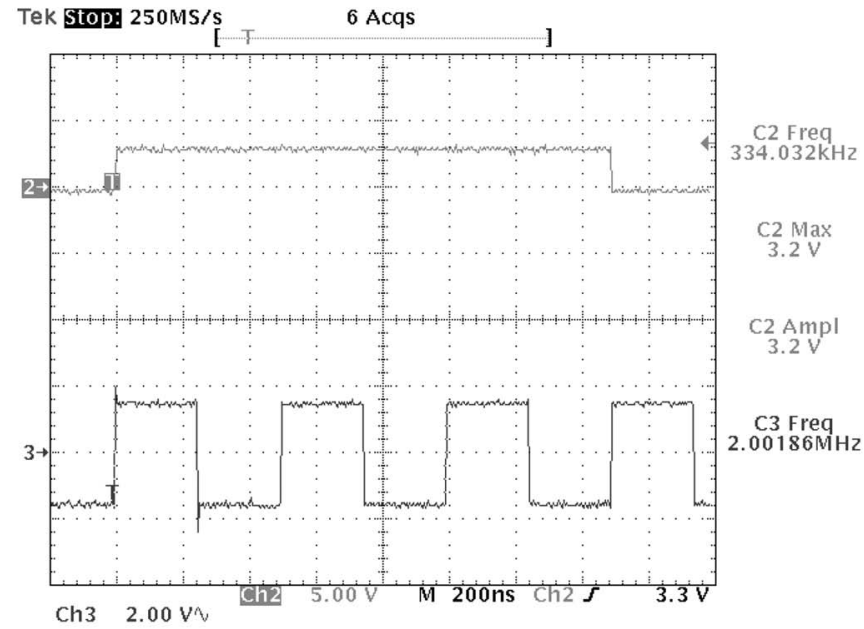

(f)

Fig. 29. Measured results of the proposed autocompensated sensor transducer under the capacitance $C_{x}$ of (a) $6.6 \mathrm{pF},(\mathrm{b}) 8 \mathrm{pF},(\mathrm{c}) 12 \mathrm{pF},(\mathrm{d}) 16 \mathrm{pF}$, (e) $20 \mathrm{pF}$, and (f) $22 \mathrm{pF}$.

the proposed autocompensated sensor transducer applied on the air pressure sensor. The applied scale of the air pressure ranges from 0.5 to $6 \mathrm{lb} / \mathrm{in}^{2}$. Although some specific applications need the larger operating range of the air pressure, the applied scale of $6 \mathrm{lb} / \mathrm{in}^{2}$ is still suitable in the wireless microsystem, such as the system specification in [3]. Measurement results have successfully verified the functions and the performance of the proposed autocompensated sensor transducer and confirmed that it is possible to apply it to the air pressure sensor. The characteristics of the proposed autocompensated sensor converter for capacitive measuring systems are summarized in Table III. 


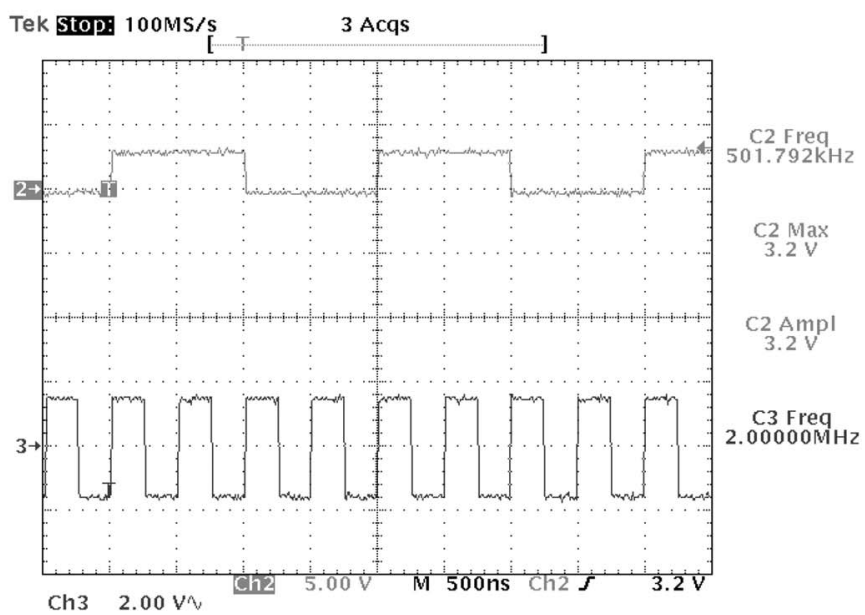

$(\mathrm{g})$

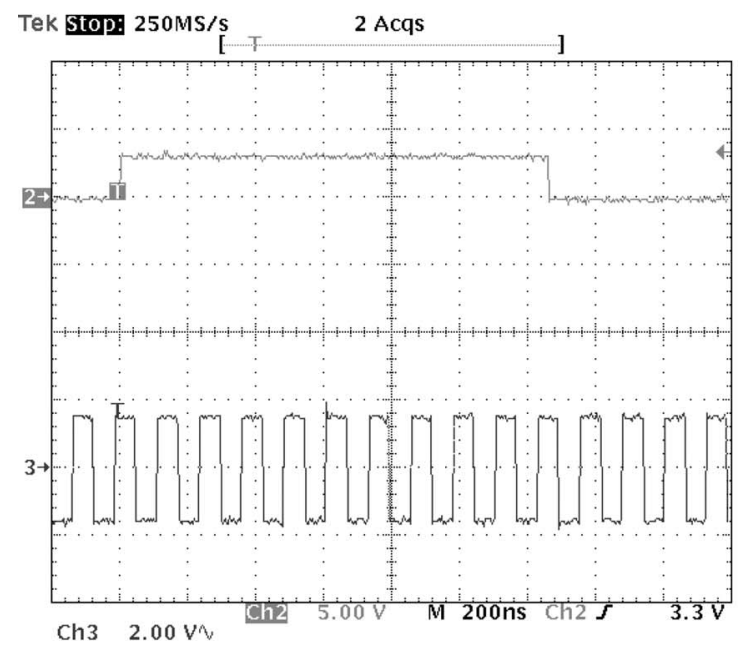

(i)

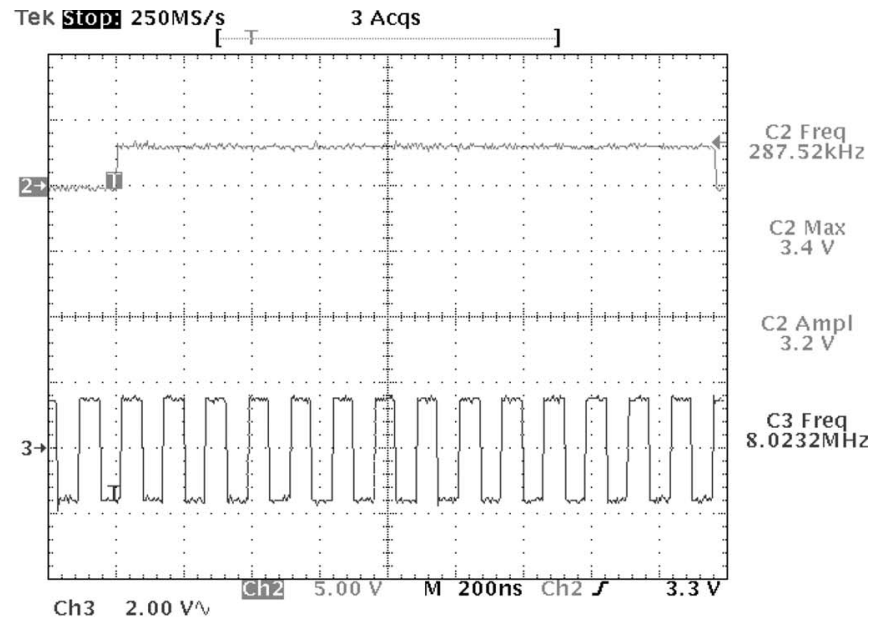

(h)

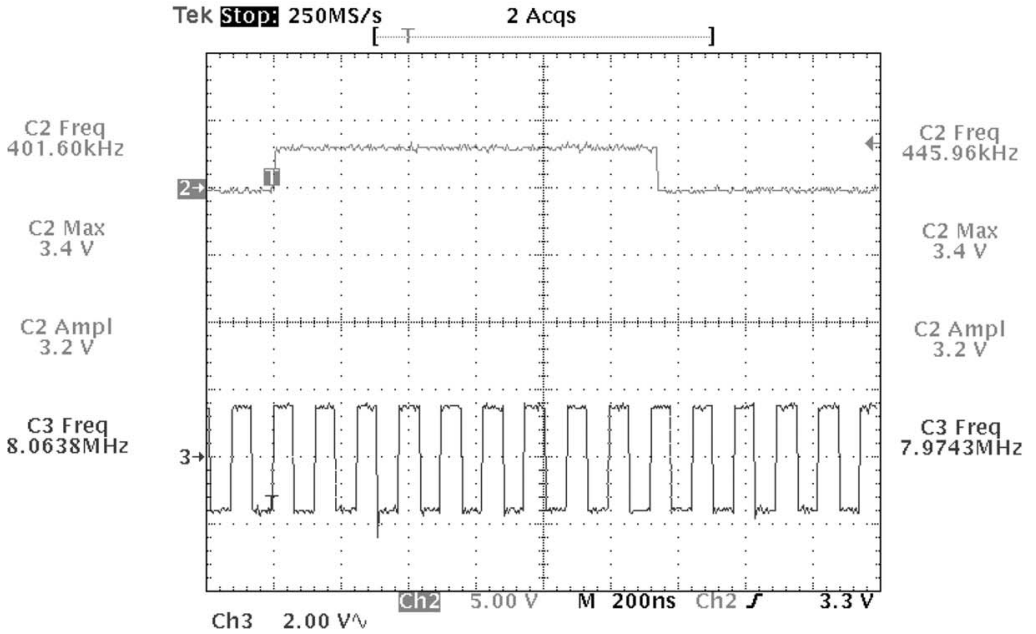

(j)

Fig. 29. (Continued.) Measured results of the proposed autocompensated sensor transducer under the capacitance $C_{x}$ of (g) $24 \mathrm{pF}$, (h) $16 \mathrm{pF}$ with compensation, (i) $20 \mathrm{pF}$ with compensation, and (j) $22 \mathrm{pF}$ with compensation.

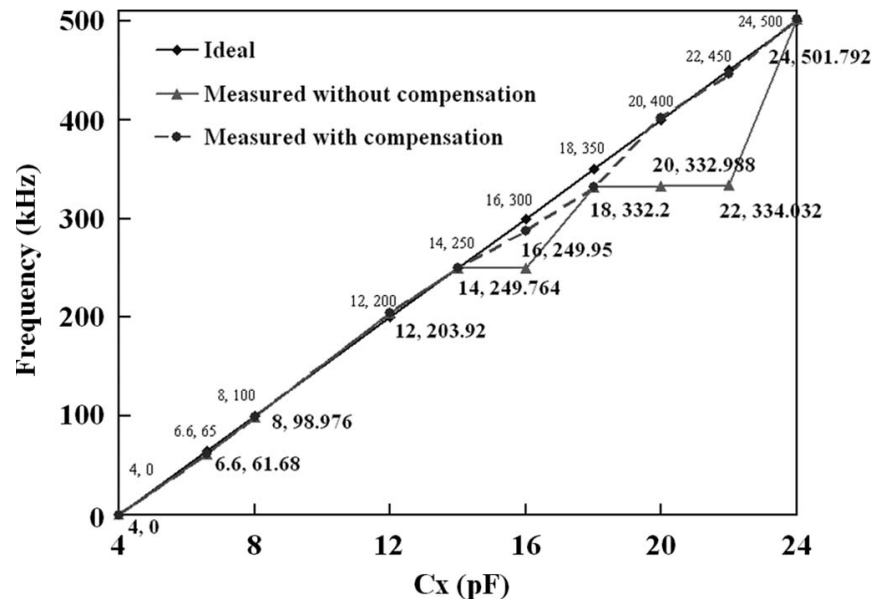

Fig. 30. Measured results of the proposed autocompensated sensor transducer under the varied capacitance of $C_{x}$, which ranges from 4 to $24 \mathrm{pF}$. The boldface-type numbers are the measured values without compensation, and the fine-type numbers are the ideal values.

\section{CONCLUSION}

A monolithic CMOS autocompensated sensor transducer for capacitive measuring systems is newly proposed. The proposed autocompensated sensor transducer is attractive due to the fact that a digitized signal is produced without realizing the analog-to-digital converter. Hence, the hardware cost can be reduced. Moreover, the output signal of the proposed transducer can easily be received and processed by the receiver terminal. The automatic compensation circuits enhance and compensate the linear relation between the variable capacitance of the detected sensor and the output digital frequency over the wide dynamic frequency range. In the measurement results, all the functions and the performance of the proposed autocompensated sensor transducer have been successfully verified, and it has been confirmed that it is possible to apply the transducer on the air pressure sensor. In future research, the proposed autocompensated sensor transducer will be integrated with the tire pressure gauge to detect tire pressure, and the pressure information will then be transmitted through a wireless sensor network.

\section{ACKNOWLEDGMENT}

The authors would like to thank the SoC Technology Center, Industrial Technology Research Institute, Hsinchu, Taiwan, 


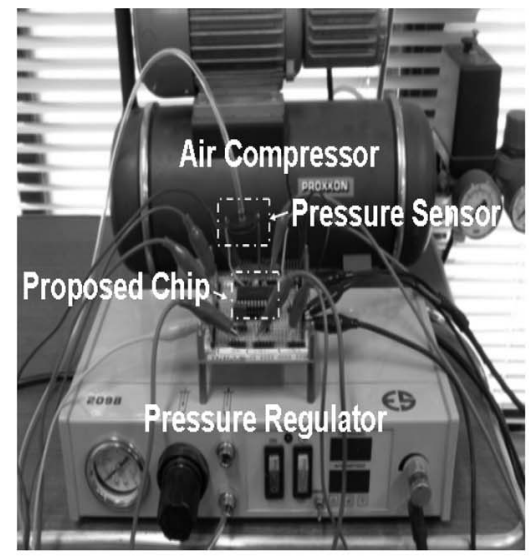

(a)

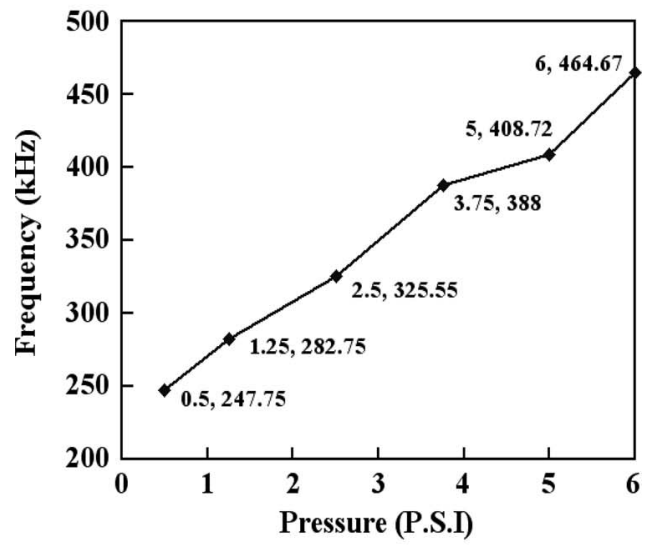

(b)

Fig. 31. (a) Whole measurement setup. (b) Measured results of the proposed autocompensated sensor transducer applied on the air pressure sensors.

TABLE III

SUMMARY ON THE CHARACTERISTICS OF A MONOLITHIC CMOS AUTOCOMPENSATED SENSOR TRANSDUCER FOR Capacitive Measuring Systems

\begin{tabular}{c|c}
\hline Technology & $0.35 \mu \mathrm{m}$ CMOS 2P4M \\
\hline Methodology of the interface circuit & Switched-capacitor technique \\
\hline Power supply & $3.2 \mathrm{~V}$ \\
\hline System clock rate & $2 \mathrm{MHz}$ \\
\hline Input Capacitance range & $4-24 \mathrm{pF}$ \\
\hline Output Frequency range & $0.5-500 \mathrm{kHz}$ \\
\hline Power consumption & $6.4 \mathrm{~mW}$ \\
\hline Accuracy & $\pm 5.14 \%$ \\
\hline Physical layout area & $940 \times 1080 \mu \mathrm{m}^{2}$ \\
\hline Application field & Sensor interface \\
\hline
\end{tabular}

R.O.C., for their support in chip fabrication and Sinopulsar Technology Inc., Hsinchu, for their support with the pressure sensor.

\section{REFERENCES}

[1] L. K. Baxter, Capacitive Sensors Design and Applications. New York: IEEE Press, 1997.

[2] S. Chatzandroulis, D. Tsoukalas, and P. A. Neukomm, "A miniature pressure system with a capacitive sensor and a passive telemetry link for use in implantable applications," J. Microelectromech. Syst., vol. 9, no. 1, pp. 18-23, Mar. 2000.

[3] A. D. DeHennis and K. D. Wise, "A wireless microsystem for the remote sensing of pressure, temperature, and relative humidity," J. Microelectromech. Syst., vol. 14, no. 1, pp. 12-22, Feb. 2005.
[4] Y. Wang, M. Nishikawa, R. Maeda, M. Fukunaga, and K. Watanabe, "A smart thermal environment monitor based on IEEE 1451.2 standard for global networking," IEEE Trans. Instrum. Meas., vol. 54, no. 3, pp. 1321-1326, Jun. 2005.

[5] K. Watanabe, H. Matsumote, and K. Fujiwara, "Switched-capacitor frequency-to-voltage and voltage-to-frequency converters," IEEE Trans. Circuits Syst., vol. CAS-33, no. 8, pp. 836-838, Aug. 1986.

[6] H. Matsumoto and K. Watanabe, "Switched-capacitor frequency-to-voltage and voltage-to-frequency converters based on charge balancing principle," in Proc. IEEE Int. Symp. Circuits Syst., 1988, pp. 2221-2224.

[7] D. Yin, Z. Zhang, and J. Li, "A simple switched-capacitor-based capacitance-to-frequency converter," Analog Integr. Circuits Signal Process., vol. 1, no. 4, pp. 353-361, Dec. 1991.

[8] F. Krummenacher, "A high-resolution capacitance-to-frequency converter," IEEE J. Solid-State Circuits, vol. SSC-20, no. 3, pp. 666-670, Jun. 1985.

[9] C.-T. Chiang, C.-S. Wang, and Y.-C. Huang, "A CMOS integrated capacitance-to-frequency converter with digital compensation circuit designed for sensor interface applications," in Proc. IEEE Int. Conf. Sensors, Oct. 2007, pp. 954-957.

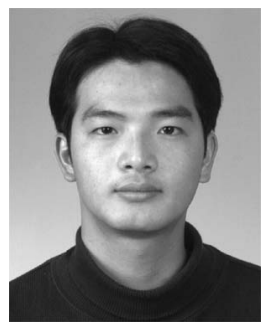

Cheng-Ta Chiang (S'00-M'05) was born in Taiwan, R.O.C., in 1977. He received the B.S. degree in electronics engineering from Chung Yuan Christian University, Jhongli, Taiwan, in 1999, the M.S. degree in biomedical engineering from the National Cheng Kung University, Tainan, Taiwan, in 2001, and the Ph.D. degree in electronics engineering from the National Chiao Tung University, Hsinchu, Taiwan, in 2006.

He was a Visiting Scholar with the Department of Electrical and Computer Engineering, The Johns Hopkins University, Baltimore, MD, from October 1, 2004 until November 30, 2005. He was included in Marquis Who's Who in Science and Engineering 2006-2007 and Marquis Who's Who in the World 2008. He was a review committee member of the National Chip Implementation Center, Hsinchu. He is currently with Mixed Signal Design Technologies Division, SoC Technology Center, Industrial Technology Research Institute, Hsinchu. His main research interests include analog integrated circuits, biomedical electronics, image sensor circuits and systems, sensor signal conditioning and transducers, and Nyquist A/D converters.

Dr. Chiang is a journal reviewer for the IEEE TRANSACTIONS ON INSTRUMENTATION AND MEASUREMENT and the IEEE SENSORS JOURNAL.

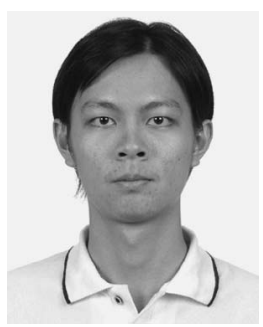

Chi-Shen Wang was born in Taiwan, R.O.C., in 1982. He received the B.S. degree in electronics engineering from the National Chung Cheng University, Chiayi, Taiwan, in 2004 and the M.S. degree in electronics engineering from the National Chiao Tung University, Hsinchu, Taiwan, in 2006.

$\mathrm{He}$ is currently with Mobile Devices Incorporation, Hsinchu, and also with the Measurement Techniques Laboratory, Department of Electronics Engineering and Institute of Electronics, National Chiao Tung University. His main research interests include analog integrated circuits, pipelined A/D converters, and sensor converters.

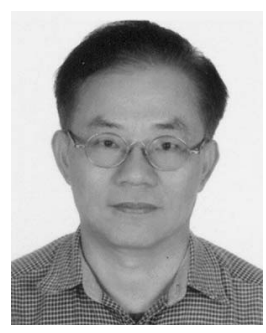

Yu-Chung Huang received the M.S. degree in electrical engineering and the Ph.D. degree in process engineering from the Technology University of Berlin, Berlin, Germany, in 1982 and 1985, respectively.

Since 1985, he has been a Professor with the Department of Electronics Engineering and Institute of Electronics, National Chiao Tung University, Hsinchu, Taiwan, R.O.C. His research interests include sensors and measuring technologies.

Dr. Huang is a member of the Committee of the Chinese Metrology Society and a member of the Micromechanical Science Institute, Taiwan. 